\title{
A new solution method for free vibration analysis of rectangular laminated composite plates with general stacking sequences and edge restraints
}

\author{
Maryam Abedi ${ }^{\mathrm{a}}$, Ramazan-Ali Jafari-Talookolaei ${ }^{\mathrm{b}, *}$, Paolo S.Valvo ${ }^{\mathrm{c}}$ \\ ${ }^{a}$ Department of Engineering and Technology, University of Mazandaran, Babolsar, Iran \\ ${ }^{\mathrm{b}}$ School of Mechanical Engineering, Babol Noshirvani University of Technology, Shariati Av., 47148-71167, Babol, \\ Mazandaran, Iran \\ ${ }^{\mathrm{c}}$ Department of Civil and Industrial Engineering, University of Pisa, Largo Lucio Lazzarino, I-56122 Pisa, Italy
}

\begin{abstract}
A method is presented to study the free vibrations of rectangular laminated composite plates with general layups and arbitrary boundary conditions. Based on the first-order shear deformation theory, the governing differential equations and boundary conditions are deduced via Hamilton's principle. Generalised displacements are expanded as series with Legendre polynomials as a base functions. A generalised eigenvalue problem is obtained by following a variational approach, where energy functional is extremised and boundary conditions are introduced by means of Lagrange multipliers. In order to overcome some difficulties in obtaining the natural frequencies and corresponding mode shapes, a new numerical strategy is proposed.
\end{abstract}

Keywords: Laminated composite plate; Free vibration; Analytical solution; Legendre polynomials; Lagrange multipliers.

\section{Introduction}

Laminated plates made of fibre-reinforced composite materials are used extensively in modern engineering - especially, in the aerospace, civil, mechanical, and nuclear industries- due to their excellent mechanical performances. Through proper arrangement of the stacking sequence (i.e., the thickness, material properties, and fibre orientation of each constituent lamina), the strength and stiffness of a laminated plate can be tailored to satisfy the given design requirements. Nonetheless, when general (unsymmetric) stacking sequences are used, various coupling effects - such as bending-extension, bending-twisting, and extension-twisting couplings-may appear, because of the overall anisotropy of the laminated plate. Such effects alter the elastic behaviour and complicate the structural analysis with respect to homogeneous and isotropic plates [1].

On one hand, it is therefore very important to have a good understanding of the peculiarities of the mechanical behaviour of laminated composite plates. On the other hand, specialised, effective, and reliable tools are necessary for both the static and dynamic analyses of such composite structures. Various analytical solutions and numerical methods for the analysis of laminated composite plates are available in the literature. Recently, Sayyad and Ghugal [2] have presented a review article on the methods for the vibration analysis of laminated composite and sandwich plates. This problem has been studied intensively during the last decades. However, most studies are confined to special cases, such as cross-ply and angle-ply laminates, symmetric or anti-symmetric stacking sequences, and specific boundary conditions. Only a few studies concern laminated plates with general stacking sequences and elastic couplings, for which the governing differential equations are highly coupled and difficult to be solved. Moreover, as pointed out by many researchers, since composite laminates have very low transverse shear modulus compared to their in-plane elastic modulus, classical lamination theory may not be adequate even for the analysis of plates with high span-to-thickness ratios. Thus, shear deformation is another important aspect in the analysis of laminated composite plates.

A number of plate theories accounting for shear deformation have been proposed. In the simplest formulations, a constant transverse displacement in the thickness direction is considered and thus the transverse normal strain and stress are neglected. According to the literature, this assumption leads to accurate results only if the analysed plates have a sufficiently large side-to-thickness ratio. Instead, for lower values of the abovementioned ratio, the contribution of the transverse normal strain and stress should be considered to have more accurate results. Among the shear deformation theories in which the abovementioned strain and stress are not neglected, we recall the pioneering works by Lo et al. [3, 4] and Kant and Owen [5, 6]. Batra and Vidoli [7] have developed a three-dimensional mixed variational principle to derive a $\mathrm{K}^{\mathrm{th}}$-order two-dimensional linear theory for an anisotropic homogeneous piezoelectric plate. The mechanical and electric displacements, the electric potential, and the in-plane components of the stress tensor are expressed as a finite series of order $\mathrm{K}$ in the thickness coordinate. Besides, the transverse normal and shear stresses, and the transverse 
electric displacement are expressed as a finite series of order $(\mathrm{K}+2)$ in the thickness coordinate. Carrera and Kroplin [8] have presented another way to consider the transverse normal strain and stress in the analysis of multilayered plates. An exhaustive review all of the higher-order shear deformation theories would however be too extensive and fall out of the scopes of the present paper. The interested reader can also refer to the theories presented by Librescu et al. [9], Reddy [10], and Fiedler et al. [11]. In what follows, we limit ourselves to cite a selection of papers that are directly related to the present work. Some of the cited theories will be used also for the comparison of results presented later on. Aghababaei and Reddy [12] have reformulated the well-known shear deformation plate theory of Reddy [10] by using the non-local linear elasticity theory. They have analysed the bending and vibration behaviour of rectangular plates via Navier's method, which however can be used only for simply supported edges. Kant and Swaminathan [13], Robaldo et al. [14], Rao et al. [15], and Swaminathan and Patil [16] have carried out the free vibration analysis of laminated composite and sandwich plates based on shear deformation theories by using the finite element method (FEM). Also, Moita et al. [17] have investigated the buckling and free vibrations of laminated plates of arbitrary geometry and layups using a discrete model based on single-layer higher-order shear deformation theory. Their model was implemented through an eight-node $C^{0}$ serendipity finite element with 10 degrees of freedom per node to contemplate general applications. Park et al. [18] have used the FEM to study the effects of skew angle and stacking sequence on the dynamic response of laminated skew plates. Free vibration analysis of symmetrically laminated, rectangular plates with clamped boundary conditions has been studied by using the hierarchical finite element method by Han and Petyt [19]. Rodrigues et al. [20] have combined the cell-based finite element method with the 4-noded quadrilateral mixed interpolation of tensorial components technique to study the static and dynamic response of laminated composite plates within Carrera's unified formulation (CUF) [21].

Lanhe et al. [22] have presented a novel numerical solution technique, called the moving least squares differential quadrature method, to study the free vibration problem of generally laminated plates based on first-order shear deformation theory. Recently, Ferreira et al. [23] have used CUF and the generalised differential quadrature technique to predict the static deformation and free vibration behaviour of thin and thick isotropic and cross-ply laminated plates. Ngo-Cong et al. [24] have proposed a one-dimensional integrated radial basis function collocation technique for the free vibration analysis of laminated composite plates using first-order shear deformation theory.

The Rayleigh-Ritz method is one of the most popular methods to obtain approximate solutions for the vibrational characteristics of laminated composite rectangular plates [25-27]. Baharlou and Leissa [28] have used this method for the analysis of vibration and buckling of generally laminated plates with various boundary conditions. Aydogdu and Timarci [29] have used the Rayleigh-Ritz method to carry out the free vibration analysis of cross-ply laminated square plates with different sets of boundary conditions. Hu et al. [30] have applied this method for the vibration of angle-ply laminated plates based on Mindlin's thick plate theory. Afterwards, Lee et al. [31] have employed the same method for the free vibration analysis of symmetrically laminated composite sandwich plates with elastic edge restraints. Khorshidi and Farhadi [32] have used the Rayleigh-Ritz method to investigate the hydrostatic vibrations of a laminated composite rectangular plate partially contacting with a bounded fluid.

In this paper, a new method is presented for the free vibration analysis of rectangular laminated composite plates with general stacking sequences. Fictitious elastic restraints are introduced on the plate's four edges, allowing any particular restraint condition to be simulated. With reference to the above discussion about shear deformation theories, it should be noted that the present formulation is based on first-order shear deformation theory, which is deemed sufficient to predict the vibrational characteristics of moderately thick plates. This choice is taken here for the sake of simplicity. However, the validity of such assumption will be assessed by comparing our results with those obtained by more accurate theories. Accordingly, the plate's displacement field is expressed in terms of the mid-plane displacements and rotations. The governing differential equations and boundary conditions are deduced via Hamilton's principle. Then, to determine the plate's natural frequencies and mode shapes, the generalised displacements are expanded as series with Legendre polynomials as a base functions. Thanks to the orthogonality of such polynomials, simple expressions are obtained for the plate's kinetic and strain energy. Subsequently, a generalised eigenvalue problem is obtained by following a variational approach, where an energy functional is extremised and the boundary conditions are introduced by means of Lagrange multipliers. The main advantage of using the method of Lagrange multipliers is that the assumed displacement functions do not have to satisfy a priori the boundary conditions of the problem. Lastly, several examples of plates with different stacking sequences and boundary conditions (clamped, simply supported, and free edges) are presented to demonstrate the effectiveness and accuracy of the method.

\section{Formulation of the problem}

\subsection{Governing differential equations and boundary conditions}

Let us consider a rectangular, laminated plate of uniform thickness $h$, length $a$, and width $b$, as shown in Fig. 1 . A Cartesian reference system $O x y z$ is fixed with the origin $O$ at one of the plate's corners on the mid-plane, the $x$ - and $y$ axes respectively aligned with the plate's longitudinal and transverse directions, and the $z$-axis completing the righthanded reference system. The laminate is made of many unidirectional fibre-reinforced laminae (or plies) stacked up in different orientations with respect to the reference axes.

Moving on to the boundary conditions, we assume that elastic restraints are present on the plate's contour. We denote with $k_{d}^{x=0}, k_{d}^{x=a}, k_{d}^{y=0}$, and $k_{d}^{y=b}$ the constants of the distributed elastic springs acting on the plate's four edges for each of 
the five local degrees of freedom $d \in\{u, v, w, \phi, \psi\}$. It should be noted that such general boundary conditions allow single degrees of freedom to be free or fixed, respectively by letting $k_{d}=0$ or $k_{d} \rightarrow+\infty$. As a result, cases of clamped, simply supported, and free - as well as elastically restrained - edges can be considered by the method, as the examples in Section 3 will show. We denote with $u, v$, and $w$ the mid-plane displacements in the $x$-, $y$-, and $z$-directions, respectively; $\phi$ and $\psi$ are the angles of rotations of the normal to the mid-plane about the $x$ - and $y$-axes, respectively.

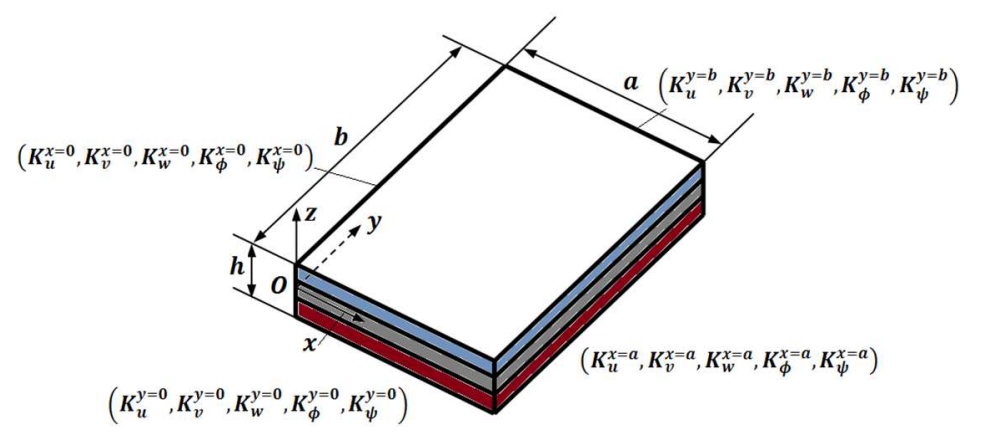

Fig. 1: Rectangular laminated composite plate with elastic edge restraints

The equations of motion and boundary conditions for the problem are derived by using Hamilton's principle as applied to a conservative system:

$\int_{t_{1}}^{t_{2}} \delta\left(T-U_{P}-U_{E}\right) d t=0$

where $T$ is the kinetic energy of the plate; $U_{P}$ and $U_{E}$ are the strain energies stored in the laminated plate and elastic edge supports, respectively; $t_{1}$ and $t_{2}$ are two arbitrary instants of time $t ; \delta$ denotes the first variation. The complete expressions of the above stated energies are presented in Appendix A. By substituting Eqs. (A.3)-(A.6) into (1) and calculating the first variation, we obtain the following set of governing partial differential equations for the motion of the laminated composite plate:

$N_{x, x}+N_{x y, y}=I_{0} u_{, t t}+I_{1} \phi_{, t t}$,

$N_{y, y}+N_{x y, x}=I_{0} v_{, t t}+I_{1} \psi_{, t t}$,

$Q_{x z, x}+Q_{y z, y}=I_{0} w_{, t t}$,

$M_{x, x}+M_{x y, y}-Q_{x z}=I_{1} u_{, t t}+I_{2} \phi_{, t t}$,

$M_{y, y}+M_{x y, x}-Q_{y z}=I_{1} v_{, t t}+I_{2} \psi_{, t t}$.

where $N_{x}, N_{y}$, and $N_{x y}$ are the in-plane resultant forces, $M_{x}$ and $M_{y}$ are the bending moments, $M_{x y}$ is the twisting moment, $Q_{y z}$ and $Q_{x z}$ are the resultant shear forces (per unit width); besides, $I_{0}, I_{1}, I_{2}$ are the mass moments of inertia, as defined by Eq. (A.7) in Appendix A. In Eq. (2), as well as in the following expressions, the convention is used that a subscript after a comma stands for partial differentiation with respect to that variable.

Furthermore, from Hamilton's principle we also obtain the boundary conditions (BC) at the edges $x=0$ and $x=a$,

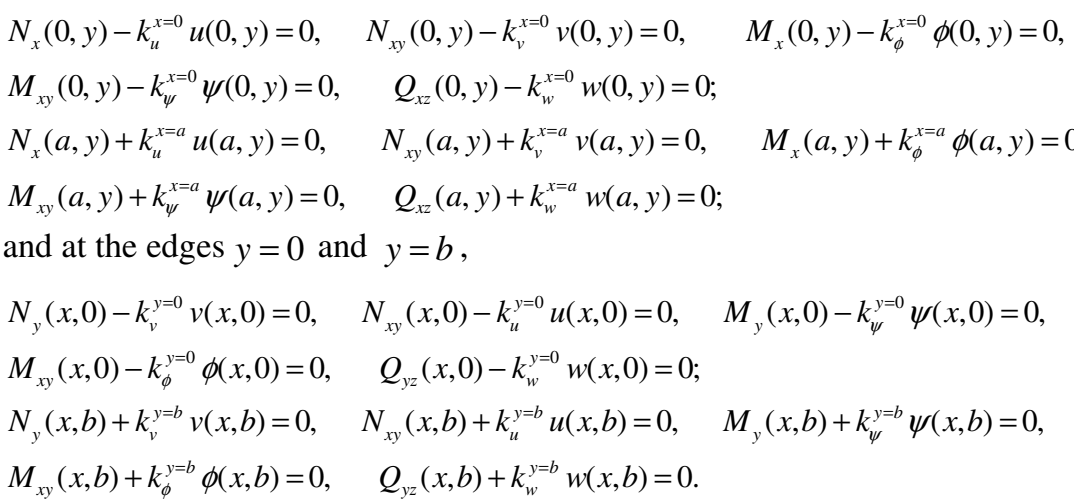

\subsection{Solution method}

To determine the natural frequencies and mode shapes of the laminated plate, we start by assuming the following harmonic solutions for the displacements:

$u(x, y, t)=U(x, y) \exp (\hat{j} \omega t), \quad v(x, y, t)=V(x, y) \exp (\hat{j} \omega t), \quad w(x, y, t)=W(x, y) \exp (\hat{j} \omega t)$,

$\phi(x, y, t)=\Phi(x, y) \exp (\hat{j} \omega t)$, and $\psi(x, y, t)=\Psi(x, y) \exp (\hat{j} \omega t)$, 
where $U(x, y), V(x, y), W(x, y), \Phi(x, y)$, and $\Psi(x, y)$ are functions of the spatial in-plane coordinates, $\omega$ is the circular frequency, and $\hat{j}=\sqrt{-1}$ denotes the imaginary unit. Next, we assume that the spatial functions can be expanded in double series as follows:

$(U(x, y), V(x, y), W(x, y), \Phi(x, y), \Psi(x, y))=\sum_{i=0}^{I} \sum_{j=0}^{J}\left(U_{i j}, V_{i j}, W_{i j}, \Phi_{i j}, \Psi_{i j}\right) P_{i}(\xi) P_{j}(\eta)$,

where $U_{i j}, V_{i j}, W_{i j}, \Phi_{i j}$ and $\Psi_{i j}$ are unknown generalised displacement amplitudes; $\xi=2 x / a-1$ and $\eta=2 y / b-1$ are non-dimensional spatial coordinates defined in the ranges $\xi \in[-1,1]$ and $\eta \in[-1,1]$, respectively; $P_{i}(\xi)$ and $P_{j}(\eta)$ respectively denote the Legendre polynomials of degrees $i$ and $j$ in the variables $\xi$ and $\eta$; $I$ and $J$ denote the number of terms that, in practice, are considered in the truncated series expansion.

By substituting the assumed solutions Eqs. (4), together with series expansions (5), into Eqs. (A.3), (A.4) and (A.6), after some simplifications, we obtain the following expressions for the strain and kinetic energies:

$$
\begin{aligned}
& U_{P}=\Re_{i m} \Re_{k m} \frac{2}{2 m+1} \frac{2}{2 j+1} \frac{b}{2 a}\left\{\begin{array}{l}
A_{11} U_{i j} U_{k j}+2 A_{16} U_{i j} V_{k j}+A_{66} V_{i j} V_{k j}+A_{55} W_{i j} W_{k j}+D_{11} \Phi_{i j} \Phi_{k j}+2 D_{16} \Phi_{i j} \Psi_{k j}+2 B_{11} \Phi_{i j} U_{k j} \\
+2 B_{16} V_{i j} \Phi_{k j}+D_{66} \Psi_{i j} \Psi_{k j}+2 B_{16} \Psi_{i j} U_{k j}+2 B_{66} \Psi_{i j} V_{k j}
\end{array}\right\} \\
& +\Re_{k i} \Re_{j l} \frac{2}{2 i+1} \frac{2}{2 l+1}\left\{\begin{array}{l}
A_{16} U_{i j} U_{k l}+A_{12} V_{i j} V_{k l}+A_{66} U_{i j} V_{k l}+A_{26} V_{i j} V_{k l}+A_{45} W_{i j} W_{k l}+D_{16} \Phi_{i j} \Phi_{k l}+D_{12} \Psi_{i j} \Phi_{k l}+B_{16} U_{i j} \Phi_{k l} \\
+B_{12} V_{i j} \Phi_{k l}+D_{66} \Phi_{i j} \Psi_{k l}+B_{16} \Phi_{i j} U_{k l}+B_{66} \Phi_{i j} V_{k l}+D_{26} \Psi_{i j} \Psi_{k l}+B_{66} U_{i j} \Psi_{k l} \\
+B_{26} V_{i j} \Psi_{k l}+B_{12} \Psi_{i j} U_{k l}+B_{26} \Psi_{i j} V_{k l}
\end{array}\right\} \\
& +\Re_{j n} \Re_{l n} \frac{2}{2 i+1} \frac{2}{2 n+1} \frac{a}{2 b}\left\{\begin{array}{l}
A_{66} U_{i j} U_{i l}+2 A_{26} V_{i j} U_{i l}+A_{22} V_{i j} V_{i l}+A_{44} W_{i j} W_{i l}+2 B_{22} \Psi_{i j} V_{i l}+D_{66} \Phi_{i j} \Phi_{i l}+2 D_{26} \Phi_{i j} \Psi_{i l} \\
+2 B_{66} \Phi_{i j} U_{i l}+2 B_{26} \Phi_{i j} V_{i l}+D_{22} \Psi_{i j} \Psi_{i l}+2 B_{26} \Psi_{i j} U_{i l}
\end{array}\right\} \\
& +\frac{2}{2 i+1} \frac{2}{2 j+1} \frac{a b}{8}\left\{A_{55} \Phi_{i j}^{2}+2 A_{45} \Phi_{i j} \Psi_{i j}+A_{44} \Psi_{i j}^{2}\right\}+\Re_{k i} \frac{2}{2 i+1} \frac{2}{2 j+1} \frac{b}{2}\left\{A_{55} \Phi_{i j} W_{k j}+A_{45} \Psi_{i j} W_{k j}\right\} \\
& +\Re_{l j} \frac{2}{2 i+1} \frac{2}{2 j+1} \frac{a}{2}\left\{A_{45} \Phi_{i j} W_{i l}+A_{44} \Psi_{i j} W_{i l}\right\}, \\
& U_{E}=\frac{b}{2} \frac{U_{i j} U_{k j}}{2 j+1}\left[k_{u}^{x=0}(-1)^{i+k}+k_{u}^{x=a}(+1)^{i+k}\right]+\frac{a}{2} \frac{U_{i j} U_{i l}}{2 i+1}\left[k_{u}^{y=0}(-1)^{j+l}+k_{u}^{y=b}(+1)^{j+l}\right] \\
& +\frac{b}{2} \frac{V_{i j} V_{k j}}{2 j+1}\left[k_{v}^{x=0}(-1)^{i+k}+k_{v}^{x=a}(+1)^{i+k}\right]+\frac{a}{2} \frac{V_{i j} V_{i l}}{2 i+1}\left[k_{v}^{y=0}(-1)^{j+l}+k_{v}^{y=b}(+1)^{j+l}\right] \\
& +\frac{b}{2} \frac{W_{i j} W_{k j}}{2 j+1}\left[k_{w}^{x=0}(-1)^{i+k}+k_{w}^{x=a}(+1)^{i+k}\right]+\frac{a}{2} \frac{W_{i j} W_{i l}}{2 i+1}\left[k_{w}^{y=0}(-1)^{j+l}+k_{w}^{y=b}(+1)^{j+l}\right] \\
& +\frac{b}{2} \frac{\Phi_{i j} \Phi_{k j}}{2 j+1}\left[k_{\phi}^{x=0}(-1)^{i+k}+k_{\phi}^{x=a}(+1)^{i+k}\right]+\frac{a}{2} \frac{\Phi_{i j} \Phi_{i l}}{2 i+1}\left[k_{\phi}^{y=0}(-1)^{j+l}+k_{\phi}^{y=b}(+1)^{j+l}\right] \\
& +\frac{b}{2} \frac{\Psi_{i j} \Psi_{k j}}{2 j+1}\left[k_{\psi}^{x=0}(-1)^{i+k}+k_{\psi}^{x=a}(+1)^{i+k}\right]+\frac{a}{2} \frac{\Psi_{i j} \Psi_{i l}}{2 i+1}\left[k_{\psi}^{y=0}(-1)^{j+l}+k_{\psi}^{y=b}(+1)^{j+l}\right], \\
& T=-\frac{a b}{8} \omega^{2}\left[I_{0}\left(U_{i j}^{2}+V_{i j}^{2}+W_{i j}^{2}\right)+2 I_{1}\left(U_{i j} \Phi_{i j}+V_{i j} \Psi_{i j}\right)+I_{2}\left(\Phi_{i j}^{2}+\Psi_{i j}^{2}\right)\right] \frac{2}{2 i+1} \frac{2}{2 j+1},
\end{aligned}
$$

where the harmonic time variation, $\exp (2 \hat{j} \omega t)$, is omitted for simplicity. In Eqs. (6), as well as in subsequent expressions, the dummy (repeated) indices imply summation. To this aim, if not otherwise specified, indices $i, k$, and $m$ range from 0 to $I$; indices $j, l$, and $n$ range from 0 to $J$. It should be noted also that, to obtain Eqs. (6), we have used the orthogonality property,

$\int_{-1}^{1} P_{i}(s) P_{j}(s) d s= \begin{cases}0, & \text { if } i \neq j, \\ \frac{2}{2 i+1}, & \text { if } i=j,\end{cases}$

and the following additional property of Legendre polynomials [33]:

$\frac{d}{d s} P_{i}(s)=\sum_{k=0}^{\left\lfloor\frac{i-1}{2}\right\rfloor}(2 i-4 k-1) P_{i-2 k-1}(s) \quad(i \geq 1)$,

where $\lfloor x\rfloor$ denotes the integer part of $x$. Eq. (8) can be rewritten as:

$\frac{d}{d s} P_{i}(s)=(2 i-1,2 i-5,2 i-9, \ldots)\left\{\begin{array}{c}P_{i-1} \\ P_{i-3} \\ P_{i-5} \\ \vdots\end{array}\right\}=(2 i-1,0,2 i-5,0,2 i-9,0, \ldots)\left\{\begin{array}{c}P_{i-1} \\ P_{i-2} \\ P_{i-3} \\ \vdots\end{array}\right\}=\sum_{m=0}^{i-1} \Re_{i m} P_{m}(s) \quad(i \geq 1)$,

out of which the coefficients $\mathfrak{R}_{i m}$ are defined for $m$ ranging from 0 to $i-1$. For $m \geq i$, we set $\mathfrak{R}_{i m}=0$.

By substituting Eqs. (4) and (5) into (A.1) and (A.5), after simplification, the first boundary condition in Eq. (3a) takes the following form:

$$
\begin{aligned}
\mathbf{C}_{1}: & \frac{2}{a} A_{11} \Re_{i m}(-1)^{m} P_{j}(\eta) U_{i j}+\frac{2}{b} A_{12}(-1)^{i} \Re_{j n} P_{n}(\eta) V_{i j}+A_{16}\left\{\frac{2}{b}(-1)^{i} \Re_{j n} P_{n}(\eta) U_{i j}+\frac{2}{a} \Re_{i m}(-1)^{m} P_{j}(\eta) V_{i j}\right\}+\frac{2}{a} B_{11} \Re_{i m}(-1)^{m} P_{j}(\eta) \Phi_{i j} \\
& +\frac{2}{b} B_{12}(-1)^{i} \Re_{j n} P_{n}(\eta) \Psi_{i j}+B_{16}\left\{\frac{2}{b}(-1)^{i} \Re_{j n} P_{n}(\eta) \Phi_{i \bar{j}}+\frac{2}{a} \Re_{i m}(-1)^{m} P_{j}(\eta) \Psi_{i j}\right\}-k_{u}^{x=0}(-1)^{i} P_{j}(\eta) U_{i j}=0 .
\end{aligned}
$$


Since Eq. (10) must be satisfied for arbitrary values of $\eta$, the following $J+1$ conditions are determined:

$$
\begin{aligned}
\mathbf{C}_{1 j}: & \frac{2}{a} A_{11} \Re_{i m}(-1)^{m} U_{i j}+\frac{2}{b} A_{12} \Re_{l j}(-1)^{i} V_{i l}+A_{16}\left\{\frac{2}{b} \Re_{l j}(-1)^{i} U_{i l}+\frac{2}{a} \Re_{i m}(-1)^{m} V_{i j}\right\}+\frac{2}{a} B_{11} \Re_{i m}(-1)^{m} \Phi_{i j} \\
& +\frac{2}{b} B_{12} \Re_{l j}(-1)^{i} \Psi_{i l}+B_{16}\left\{\frac{2}{b} \Re_{l j}(-1)^{i} \Phi_{i l}+\frac{2}{a} \Re_{i m}(-1)^{m} \Psi_{i j}\right\}-k_{u}^{x=0}(-1)^{i} U_{i j}=0, \quad j=0,1, \ldots, J .
\end{aligned}
$$

Likewise, each of the remaining 19 boundary conditions in Eqs. (3) can be expressed in a form similar to Eq. (11). In the end, we obtain $10(J+1)$ conditions $\mathbf{C}_{\alpha j}(\alpha=1,2, \ldots 10)$ on the edges $\xi=-1$ and $\xi=1$ and $10(I+1)$ conditions $\mathbf{C}_{\beta i}($ $\beta=11,12, \ldots 20$ ) on the edges $\eta=-1$ and $\eta=1$, as detailed in Appendix B.

Now, the natural frequencies and mode shapes are determined by using a procedure based on a variational principle similar to that proposed by Washizu [34]. The following functional, defined as the sum of the strain and kinetic energies of the plate, is extremised subject to the constraints given by the boundary conditions:

$F=U_{P}+U_{E}+T+\sum_{\alpha=1}^{10} \sum_{j=0}^{J} \lambda_{\alpha j} \mathbf{C}_{\alpha j}+\sum_{\beta=11}^{20} \sum_{i=0}^{I} \lambda_{\beta i} \mathbf{C}_{\beta i}$,

where $\lambda_{\alpha j}$ and $\lambda_{\beta i}$ are Lagrange multipliers. The necessary extremising conditions are:

$\frac{\partial F}{\partial U_{i j}}=\frac{\partial F}{\partial V_{i j}}=\frac{\partial F}{\partial W_{i j}}=\frac{\partial F}{\partial \Phi_{i j}}=\frac{\partial F}{\partial \Psi_{i j}}=0$

$\frac{\partial F}{\partial \lambda_{\alpha j}}=\frac{\partial F}{\partial \lambda_{\beta i}}=0, \quad$ with $i=0,1, \ldots, I ; j=0,1, \ldots, J ; \alpha=1,2, \ldots, 10 ; \beta=11,12, \ldots, 20$.

By substituting Eqs. (6), (11) and the equations in Appendix B into (12) and (13), after some simplifications, we obtain the following generalised eigenvalue problem:

$\left(\left[\begin{array}{cc}{\left[K_{11}\right]} & {\left[K_{12}\right]} \\ {\left[K_{21}\right]} & {[0]}\end{array}\right]-\omega^{2}\left[\begin{array}{cc}{\left[M_{11}\right]} & {[0]} \\ {[0]} & {[0]}\end{array}\right]\right)\left\{\begin{array}{l}\{X\} \\ \{\lambda\}\end{array}\right\}=\{0\}$,

where the vector

$\{X\}=\left(U_{00}, U_{01}, \ldots, U_{I(J-1)}, U_{I J} ; V_{00}, \ldots, V_{I J} ; W_{00}, \ldots, W_{I J} ; \Phi_{00}, \ldots, \Phi_{I J} ; \Psi_{00}, \ldots, \Psi_{I J}\right)^{\mathrm{T}}$

orderly contains the $M=5(I+1)(J+1)$ generalised displacement amplitudes and the vector $\{\lambda\}$ contains the $N=10(I+1)+10(J+1)$ Lagrange multipliers. It is noted that both the submatrices $\left[K_{11}\right]$ and $\left[M_{11}\right]$ are positive definite, symmetric, square matrices of dimensions $M \times M$, while the submatrix [ $\left.K_{12}\right]$ has dimensions $M \times N$ and is generally rectangular. It is easy to prove that $\left[K_{21}\right]$ is the transpose of $\left[K_{12}\right]$.

The solution of the eigenvalue problem defined by Eq. (14) is complicated by the presence of many null elements in the involved matrices. To overcome this difficulty, we introduce the following change of variables:

$\left\{X^{*}\right\}=\left[M_{11}\right]^{1 / 2}\{X\}$ and $\left\{\lambda^{*}\right\}=\frac{1}{L V}\{\lambda\}$

where $\left[M_{11}\right]^{1 / 2}$ is the square root of matrix $\left[M_{11}\right]$ and $L V$ is a suitably chosen, large numerical value. Then, instead of Eq. (14), we consider the following equivalent, standard eigenvalue problem:

$\left(\left[\begin{array}{cc}{\left[A_{11}\right]} & {\left[A_{12}\right]} \\ {\left[A_{21}\right]} & {[0]}\end{array}\right]-\omega^{2}\left[\begin{array}{cc}{\left[I_{11}\right]} & {[0]} \\ {[0]} & {\left[I_{22}\right]}\end{array}\right]\right)\left\{\begin{array}{l}\left\{X^{*}\right\} \\ \left\{\lambda^{*}\right\}\end{array}\right\}=\{0\}$,

where:

$\left[A_{11}\right]=\left[M_{11}\right]^{-1 / 2}\left[K_{11}\right]\left[M_{11}\right]^{-1 / 2}, \quad\left[A_{12}\right]=(L V)\left[M_{11}\right]^{-1 / 2}\left[K_{12}\right]$, and $\left[A_{21}\right]=(L V)\left[K_{21}\right]\left[M_{11}\right]^{-1 / 2}$;

$\left[I_{11}\right]$ and $\left[I_{22}\right]$ respectively are the $M \times M$ and $N \times N$ identity matrices. The equivalence between the problems defined by Eqs. (14) and (17) can be easily proved by substituting Eqs. (16) and (18) into (17). Then, by pre-multiplying the result by the following matrix:

$\left[\begin{array}{cc}{\left[M_{11}\right]^{1 / 2}} & {[0]} \\ {[0]} & \frac{1}{L V}\left[I_{22}\right]\end{array}\right]$,

we obtain:

$\left(\left[\begin{array}{cc}{\left[K_{11}\right]} & {\left[K_{12}\right]} \\ {\left[K_{21}\right]} & {[0]}\end{array}\right]-\omega^{2}\left[\begin{array}{cc}{\left[M_{11}\right]} & {[0]} \\ {[0]} & \frac{1}{(L V)^{2}}\left[I_{22}\right]\end{array}\right]\right)\left\{\begin{array}{l}\{X\} \\ \{\lambda\}\end{array}\right\}=\{0\}$,

which, for $L V \rightarrow+\infty$, becomes identical to Eq. (14). In practice, in the examples presented below, the large value has been chosen much greater than $\omega$ to satisfy the boundary conditions. The solution of the eigenvalue problem defined by Eq. (17) has been implemented into a self-developed computer program using the MATLAB software. In this way, the natural frequencies and corresponding mode shapes can be determined for any specific problem.

DOI: https://doi.org/10.1016/j.compstruc.2016.07.007 


\section{Results}

To verify the accuracy and efficiency of the proposed method, several example problems have been solved. Some relevant results are presented in the following. In all the examples, the shear correction factor is taken to be 5/6. It is assumed that all layers have the same thickness, density, and material properties. Unless mentioned otherwise, the following properties are used for the lamina in its material principal reference:

$$
\begin{aligned}
& E_{2}=9.65 \mathrm{GPa}, \quad E_{1}=25 E_{2}, \quad v_{12}=0.25, \quad v_{21}=v_{12} E_{2} / E_{1}, \\
& G_{12}=G_{13}=0.5 E_{2}, \quad G_{23}=0.2 E_{2}, \quad \rho=1389.23 \mathrm{~kg} / \mathrm{m}^{3} .
\end{aligned}
$$

In order to model conventional boundary conditions - namely, clamped (C), simply supported (S), and free (F) edges the stiffnesses of the distributed springs are taken as follows [35]:

C: $k_{u}=k_{v}=k_{w}=10^{7} \frac{E_{1}}{12\left(1-v_{12} v_{21}\right)}, k_{\phi}=k_{\psi}=10^{7} \frac{E_{1} h^{2}}{12\left(1-v_{12} v_{21}\right)}$;

S: at $\xi= \pm 1: \quad k_{u}=k_{v}=k_{w}=10^{7} \frac{E_{1}}{12\left(1-v_{12} v_{21}\right)}, \quad k_{\phi}=0, \quad k_{\psi}=10^{7} \frac{E_{1} h^{2}}{12\left(1-v_{12} v_{21}\right)}$;

at $\eta= \pm 1: \quad k_{u}=k_{v}=k_{w}=10^{7} \frac{E_{1}}{12\left(1-v_{12} v_{21}\right)}, k_{\phi}=10^{7} \frac{E_{1} h^{2}}{12\left(1-v_{12} v_{21}\right)}, k_{\psi}=0$;

$\mathrm{F}: k_{u}=k_{v}=k_{w}=k_{\phi}=k_{\psi}=0$.

In what follows, the obtained results will be presented in terms of dimensionless natural frequencies. If not otherwise specified, these are defined as $\Omega=\left(\omega a^{2} / h\right) \sqrt{\rho / E_{2}}$.

\subsection{Two-layered square plate}

As a first example, we consider a moderately thick $(a / h=10)$, two-layered square plate with stacking sequence $\left[0^{\circ} / 45^{\circ}\right]$ and clamped edges, already analysed by Lanhe et al. [22]. Table 1 shows the dimensionless natural frequencies, here defined as $\bar{\Omega}=\omega a \sqrt{\rho / E_{2}}$, for the first five normal modes, as computed by the present method. To test the convergence rate, an increasing number of terms, $I$ and $J$, has been included in the truncated series expansion Eq. (5). As the number of terms increases, the natural frequencies converge to certain limit values. The convergence rate looks better for the lower modes rather than for the higher modes. For comparison, the table shows also the results of [22], which appear to be in good agreement with those of the present method. Based on the obtained results, from here on, the number of polynomial terms in the series expansion, $I$ and $J$, will be taken equal to 13 in all the following examples.

\section{Table 1}

First five dimensionless natural frequencies, $\bar{\Omega}$, of $\mathrm{a}\left[0^{\circ} / 45^{\circ}\right]$ square plate with clamped edges $(a / h=10)$. $\left(E_{1}=25 E_{2}, G_{12}=G_{13}=0.5 E_{2}, G_{23}=0.2 E_{2}\right)$

\begin{tabular}{ccccccc}
\hline \multirow{2}{*}{ Ref. } & \multirow{2}{*}{$I \times J$} & \multicolumn{5}{c}{ Normal mode number } \\
\cline { 3 - 7 } & & 1 & 2 & 3 & 4 & 5 \\
\hline \multirow{2}{*}{ Present } & $11 \times 9$ & 1.5477 & 2.2752 & 2.9762 & 3.3202 & 3.7080 \\
& $13 \times 13$ & 1.5443 & 2.2736 & 2.9650 & 3.3133 & 3.7013 \\
Lanhe et al. [22] & $15 \times 15$ & 1.5443 & 2.2697 & 2.9641 & 3.3104 & 3.7011 \\
\cline { 2 - 7 } & & 1.5443 & 2.2697 & 2.9641 & 3.3104 & 3.7011 \\
\hline
\end{tabular}

\subsection{Cross-ply laminated square plates}

As a second example, we consider some cross-ply laminated square plates with simply supported edges, already analysed by many authors [20, 23, 36-41]. It is worth mentioning that in references [38-41] the transverse normal strain and stress are neglected, whereas in references [20, 23, 36, 37] the influence of these factors is considered. Table 2 shows the dimensionless fundamental frequency, $\Omega$, of a plate with symmetric stacking sequence $\left[0^{\circ} / 90^{\circ} / 90^{\circ} / 0^{\circ}\right]$ for $E_{1} / E_{2}=40$ and several values of the span-to-thickness ratio, $a / h$. Tables 3 and 4 show the dimensionless fundamental frequency, $\Omega$, of a plate with symmetric stacking sequence $\left[0^{\circ} / 90^{\circ} / 90^{\circ} / 0^{\circ}\right]$ and antisymmetric stacking sequence $\left[0^{\circ} / 90^{\circ} / 0^{\circ} / 90^{\circ}\right]$, respectively, for $a / h=5$ and several values of the in-plane Young's moduli ratio, $E_{1} / E_{2}$. The results in Table 2 show that an excellent agreement is achieved between the present method and the cited references even for the moderately thick plates, i.e. with $a / h=10$. Nonetheless, a close inspection of the results presented in Tables 3 and 4 for a thick plate, i.e. with $a / h=5$, reveals that there are slight differences between our results and those of references [3841] (all neglecting the transverse normal and shear strain and stress) with respect to the results of the more accurate theories used in references $[23,36]$. 
Table 2

Dimensionless fundamental frequency, $\Omega$, of a $\left[0^{\circ} / 90^{\circ} / 90^{\circ} / 0^{\circ}\right]$ square plate with simply supported edges.

$\left(E_{1}=40 E_{2}, G_{12}=G_{13}=0.6 E_{2}, G_{23}=0.5 E_{2}\right)$

\begin{tabular}{ccccc}
\hline Ref. & \multicolumn{3}{c}{$a / h$} \\
\cline { 2 - 5 } & 10 & 20 & 50 & 100 \\
\hline Kant and Swaminathan [36] & 15.1048 & 17.6470 & 18.6720 & 18.8357 \\
Matsunaga [37] & 15.0721 & 17.6369 & 18.6702 & 18.8352 \\
Reddy [38] & 15.1073 & 17.6457 & 18.6934 & 18.8356 \\
Akavci [39] & 15.3684 & 17.7584 & 18.7895 & 18.8412 \\
Rodrigues et al.[20] & 15.1674 & 17.7471 & 18.6719 & 18.9561 \\
Present & 15.1056 & 17.6448 & & 18.8361 \\
\hline
\end{tabular}

Table 3

Dimensionless fundamental frequency, $\Omega$, of a $\left[0^{\circ} / 90^{\circ} / 90^{\circ} / 0^{\circ}\right]$ square plate with simply supported edges $(a / h=5)$. $\left(G_{12}=G_{13}=0.6 E_{2}, G_{23}=0.5 E_{2}\right)$

\begin{tabular}{ccccc}
\hline Ref. & \multicolumn{4}{c}{$E_{1} / E_{2}$} \\
\cline { 2 - 5 } & 10 & 20 & 30 & 40 \\
\hline Liew et al. [40] & 8.2924 & 9.5613 & 10.320 & 10.849 \\
Ferreira et al. [23] & 8.3150 & 9.5443 & 10.2689 & 10.7648 \\
Present & 8.2848 & 9.5490 & 10.3022 & 10.8278 \\
\hline
\end{tabular}

\section{Table 4}

Dimensionless fundamental frequency, $\Omega$, of a $\left[0^{\circ} / 90^{\circ} / 0^{\circ} / 90^{\circ}\right]$ square plate with simply supported edges $(a / h=5)$. $\left(G_{12}=G_{13}=0.6 E_{2}, G_{23}=0.5 E_{2}\right)$

\begin{tabular}{cccccc}
\hline \multirow{2}{*}{ Ref. } & \multicolumn{5}{c}{$E_{1} / E_{2}$} \\
\cline { 2 - 6 } & 3 & 10 & 20 & 30 & 40 \\
\hline Kant and Swaminathan[36] & 6.4319 & 8.1010 & 9.4338 & 10.2463 & 10.7993 \\
Reddy [38] & 6.5008 & 8.1954 & 9.6265 & 10.5348 & 11.1716 \\
Akavci [39] & 6.5009 & 8.1932 & 9.6209 & 10.526 & 11.161 \\
Noor [41] & 6.5455 & 8.1445 & 9.4055 & 10.1650 & 10.6798 \\
Present & 6.4935 & 8.1562 & 9.4825 & 10.4001 & 10.8653 \\
\hline
\end{tabular}

Our third example concerns moderately thick $(a / h=10)$, cross-ply laminated square plate with symmetric stacking sequence $\left[0^{\circ} / 90^{\circ} / 0^{\circ}\right]$ and various boundary conditions. Table 5 shows the dimensionless first five natural frequencies, here defined as $\Omega^{*}=\left(\Omega / \pi^{2}\right) \sqrt{12\left(1-v_{12} v_{21}\right)}$, as calculated by the present method and also reported by Lanhe et al. [22] and Liew [42]. An excellent agreement is obtained with the results of the literature, which validates the accuracy of the present solution method.

Table 5

First five dimensionless natural frequencies, $\Omega^{*}$, of a $\left[0^{\circ} / 90^{\circ} / 0^{\circ}\right]$ square plate with various boundary conditions $(a / h=$ $10),\left(E_{1}=40 E_{2}, G_{12}=G_{13}=0.6 E_{2}, G_{23}=0.5 E_{2}\right)$.

\begin{tabular}{ccccccc}
\hline \multirow{2}{*}{ BC } & Ref. & \multicolumn{5}{c}{ Normal mode number } \\
\cline { 3 - 7 } SSSS & Lanhe et al. [22] & 5.172 & 7.763 & 12.972 & 13.155 & 14.429 \\
& Liew [42] & 5.166 & 7.757 & 12.915 & 13.049 & 14.376 \\
& Present & 5.1745 & 7.7635 & 12.9338 & 13.0924 & 14.4209 \\
\hline \multirow{3}{*}{ CCCC } & Lanhe et al. [22] & 7.432 & 10.399 & 13.958 & 15.467 & 15.838 \\
& Liew [42] & 7.411 & 10.393 & 13.913 & 15.429 & 15.806 \\
& Present & 7.4132 & 10.3911 & 13.9462 & 15.4513 & 15.8305 \\
\hline \multirow{3}{*}{ CSCS } & Lanhe et al. [22] & 5.875 & 9.551 & 13.428 & 14.889 & 15.365 \\
& Liew [42] & 5.871 & 9.454 & 13.340 & 14.878 & 15.340 \\
& Present & 5.8722 & 9.2346 & 13.4212 & 14.8543 & 15.3421 \\
\hline & Lanhe et al. [22] & 2.065 & 3.945 & 7.839 & 8.621 & 8.873 \\
CCFF & Liew [42] & 2.063 & 3.947 & 7.844 & 8.580 & 8.868 \\
& Present & 2.0599 & 3.9317 & 7.8653 & 8.5750 & 8.8590 \\
\hline \multirow{3}{*}{ CFFF } & Lanhe et al. [22] & 1.919 & 2.105 & 4.175 & 7.757 & 7.996 \\
& Liew [42] & 1.918 & 2.103 & 4.188 & 7.757 & 7.961 \\
SSFF & Present & 1.8933 & 2.0867 & 4.1811 & 7.7612 & 7.9749 \\
& Lanhe et al. [22] & 0.447 & 2.675 & 7.233 & 7.320 & 8.044 \\
& Liew [42] & 0.444 & 2.671 & 7.248 & 7.316 & 8.037 \\
\hline
\end{tabular}




\subsection{Angle-ply laminated square plates}

As a fourth example, we consider an angle-ply laminated square plate with clamped (CCCC) or simply supported edges (SSSS). For each boundary condition, both symmetric $[\theta /-\theta]_{S}$ and antisymmetric $[\theta /-\theta]_{A S}$ stacking sequences have been analysed. The span-to-thickness ratio is $a / h=15$. Figures 2 and 3 display the first three dimensionless natural frequencies, $\Omega$, versus the ply orientation angle, $\theta$, as computed via the proposed method. It is observed that both the ply orientation and boundary conditions have a relevant effect on the computed natural frequencies. For the antisymmetric stacking sequences, the second and third natural frequencies for ply orientation $\theta=45^{\circ}$ are very close to each other. This type of behaviour has been also reported in references [22] and [43]. For all the analysed cases, the maximum fundamental frequency occurs for $\theta=45^{\circ}$.

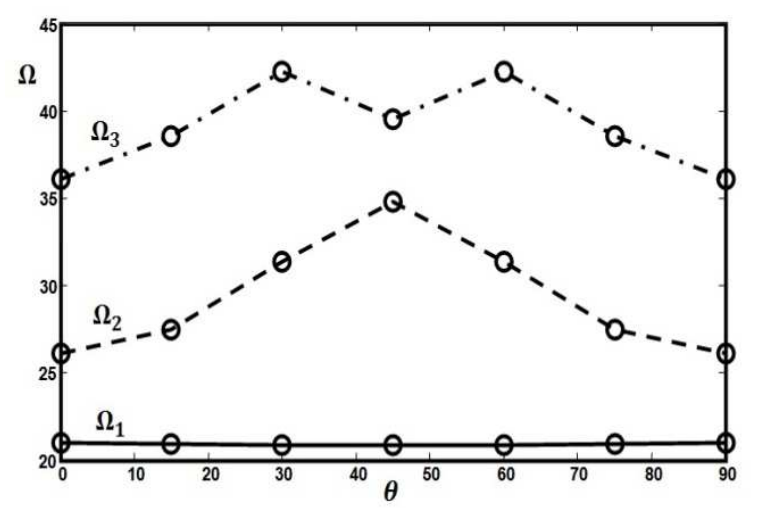

(a) Symmetric laminates

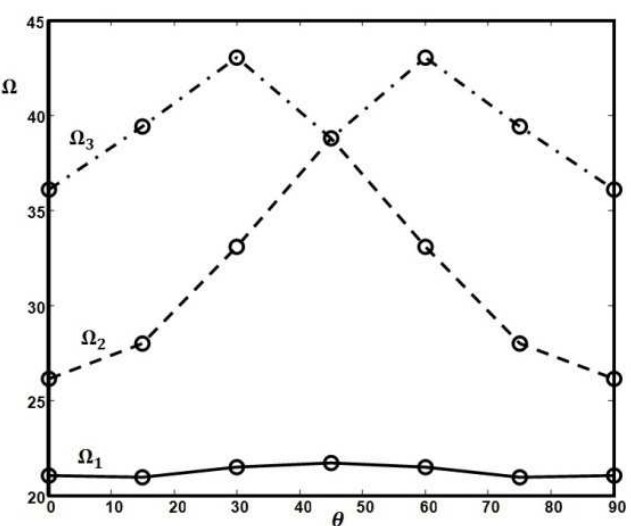

(b) Antisymmetric laminates

Fig. 2. First three dimensionless natural frequencies vs. ply orientation angle of a clamped angle-ply square plate: (a) symmetric stacking sequences $[\theta /-\theta]_{S} ;(\mathrm{b})$ antisymmetric stacking sequences $[\theta /-\theta]_{A S}$.

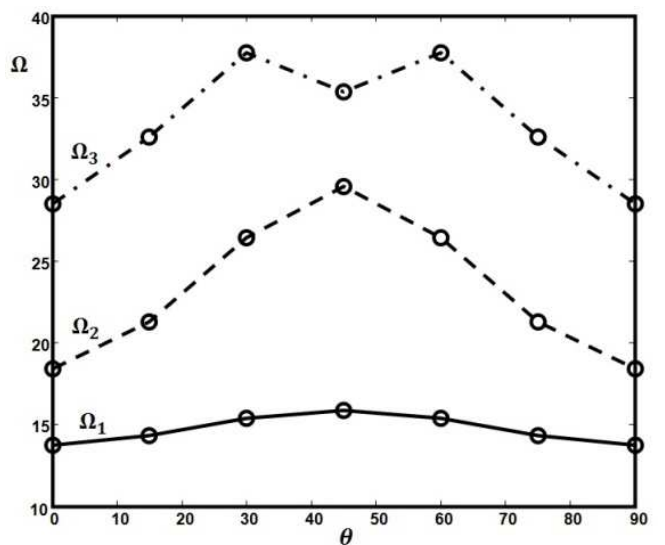

(a) Symmetric laminates

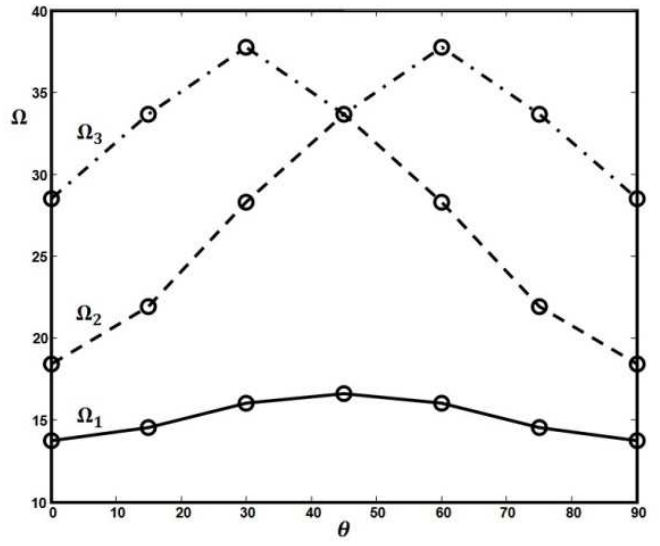

(b) Antisymmetric laminates

Fig. 3. First three dimensionless natural frequencies vs. ply orientation angle of a simply supported angle-ply square plate: (a) symmetric stacking sequences $[\theta /-\theta]_{S}$; (b) antisymmetric stacking sequences $[\theta /-\theta]_{A S}$.

\subsection{Unsymmetric laminated square plates}

Our fifth example concerns a thick $(a / h=15)$, square plate with unsymmetric stacking sequence $\left[0^{\circ} / 30^{\circ} / 60^{\circ} / 0^{\circ}\right]$ and various boundary conditions. The first six dimensionless natural frequencies, $\Omega$, are given in Table 6 . The results show that the boundary conditions have a significant effect on the vibrational characteristics of the plate. In particular, the CCCC and SSFF boundary conditions correspond to the higher and lower values of the natural frequencies, respectively. Figure 4 illustrates the mode shapes of the considered plate with clamped edges (CCCC) corresponding to the first six natural frequencies. 
Table 6

First six dimensionless natural frequencies, $\Omega$, of a $\left[0^{\circ} / 30^{\circ} / 60^{\circ} / 0^{\circ}\right]$ square plate with various boundary conditions $(a / h$ $=15)$.

\begin{tabular}{ccccccc}
\hline Boundary & \multicolumn{5}{c}{ Mode sequence number } \\
\cline { 2 - 7 } Conditions & 1 & 2 & 3 & 4 & 5 & 6 \\
\hline CCCC & 22.4671 & 29.5356 & 42.1175 & 45.8390 & 50.9737 & 57.2457 \\
CSCS & 21.4314 & 26.1045 & 36.9858 & 45.2949 & 49.0023 & 52.1889 \\
SSSS & 14.0078 & 21.1871 & 34.2656 & 40.0709 & 44.7157 & 50.3551 \\
CCFC & 9.3382 & 20.4722 & 25.2288 & 32.4470 & 36.5999 & 45.1826 \\
CCFF & 4.9942 & 9.5360 & 20.7957 & 22.8279 & 26.4994 & 33.7152 \\
SSFS & 4.6467 & 14.3031 & 20.8070 & 26.4893 & 30.3359 & 38.8619 \\
CFFF & 4.5492 & 5.5872 & 10.7881 & 21.7276 & 22.4729 & 23.8215 \\
SSFF & 1.6000 & 6.8903 & 17.5510 & 19.0985 & 22.9712 & 29.9500 \\
\hline
\end{tabular}

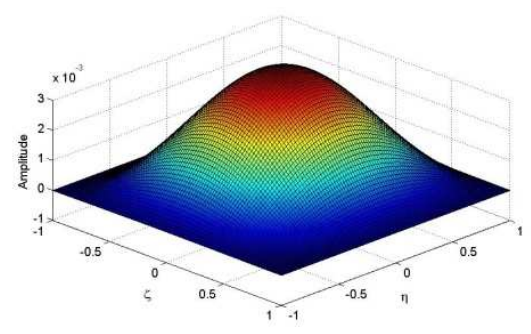

(a) First mode

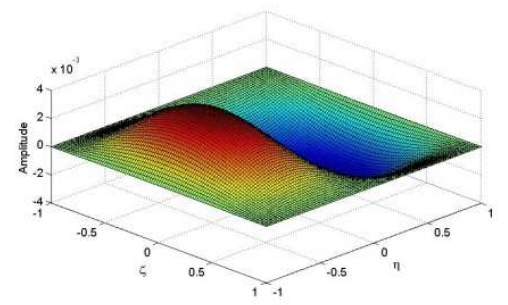

(d) Fourth mode

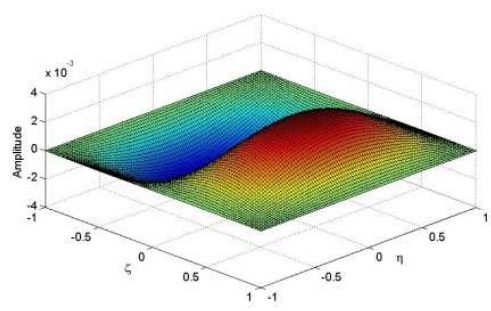

(b) Second mode

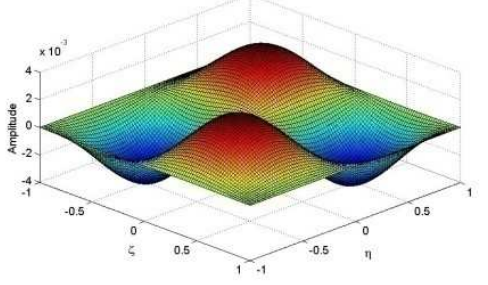

(e) Fifth mode

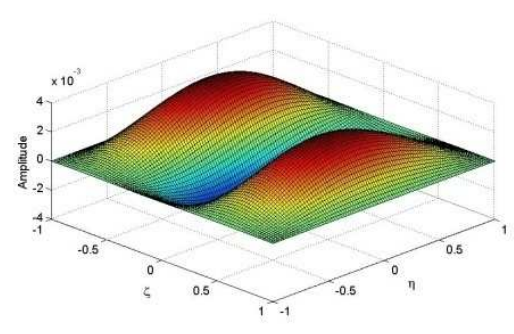

(c) Third mode

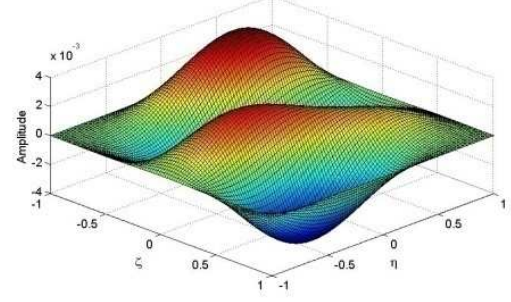

(f) Sixth mode

Fig. 4. First six mode shapes of a $\left[0^{\circ} / 30^{\circ} / 60^{\circ} / 0^{\circ}\right]$ square plate with clamped edges $(a / h=15)$.

As a sixth example, we consider a square plate with unsymmetric stacking sequence $\left[0^{\circ} / 50^{\circ} / 40^{\circ} / 0^{\circ}\right]$ and clamped (CCCC) or simply supported edges (SSSS). The first three dimensionless natural frequencies, $\Omega$, as functions of the span-to-thickness ratio, $a / h$, are plotted in figure 5. For both boundary conditions, as the span-to-thickness ratio increases, the computed natural frequencies increase and approach the values predicted by classical lamination theory (without shear deformability).

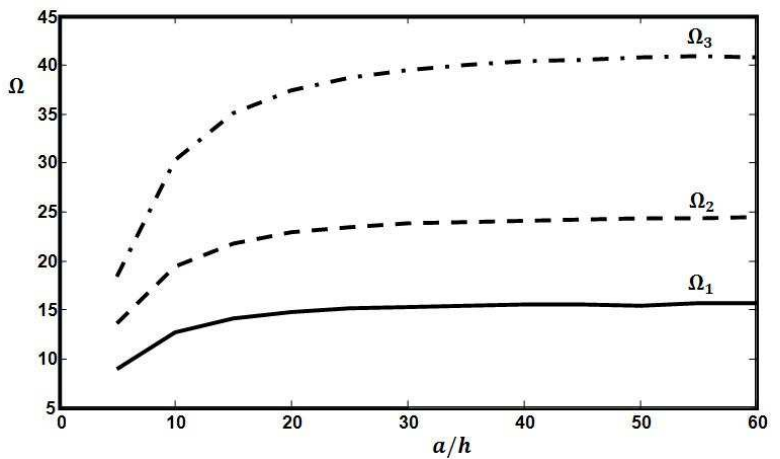

(a) SSSS

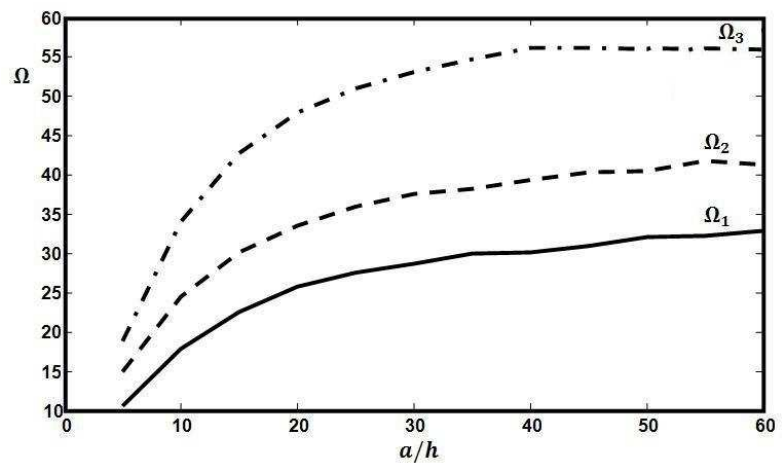

(b) $\mathrm{CCCC}$

Fig. 5. First three dimensionless natural frequencies vs. span-to-thickness ratio of $\left[0^{\circ} / 50^{\circ} / 40^{\circ} / 0^{\circ}\right]$ square plate: (a) simply supported edges; (b) clamped edges.

\subsection{Symmetric cross-ply rectangular plate}

The seventh example concerns a thick $(a / h=10)$ rectangular plate with stacking sequence $\left[0^{\circ} / 90^{\circ}\right]_{2 s}$. Figure 6 shows the first three dimensionless natural frequencies, $\Omega$, as functions of the aspect ratio of the plate, $b / a$, in the case of simply supported edges (SSSS). As the aspect ratio of the plate increases, the natural frequencies decrease. This effect appears to be less pronounced for the larger values of the aspect ratio. 


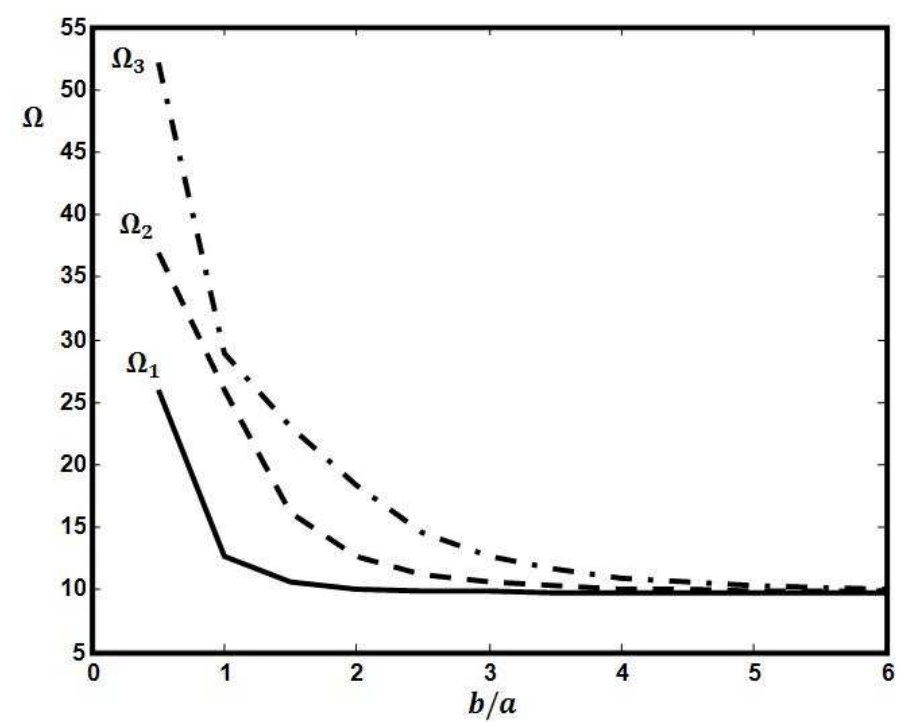

Fig. 6. First three dimensionless natural frequencies vs. aspect ratio of a $\left[0^{\circ} / 90^{\circ}\right]_{2 S}$ simply supported rectangular plate $(a / h=10)$.

In addition, the square plate with the same stacking sequence has been analysed by assuming CSCS and CCFC boundary conditions. For such cases, figure 7 shows the first three dimensionless natural frequencies, $\Omega$, as functions of the in-plane Young's moduli ratio, $E_{1} / E_{2}$. The computed natural frequencies increase as the material anisotropic ratio increases. This effect appears to be more pronounced for the higher modes.

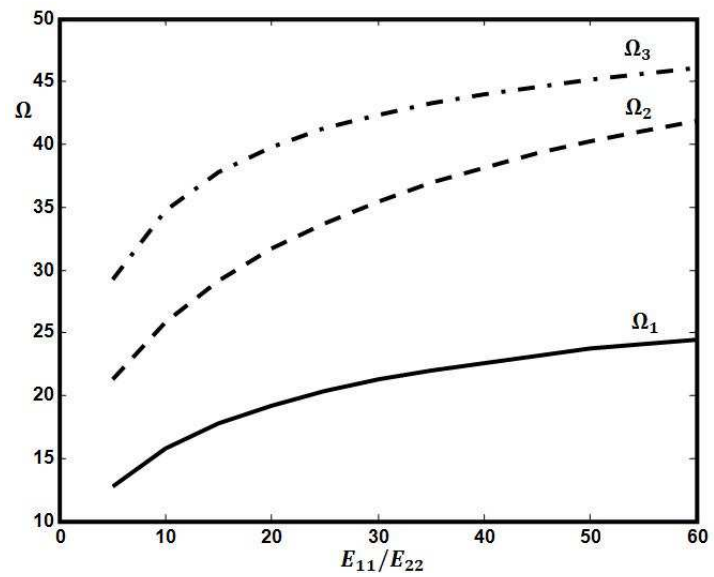

(a) CSCS

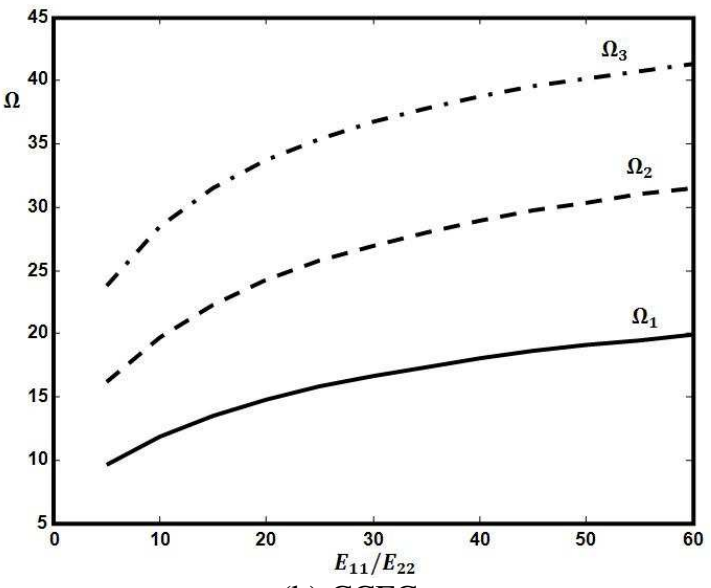

(b) $\mathrm{CCFC}$

Fig. 7. First three dimensionless natural frequencies vs. in-plane Young's moduli ratio of a $\left[0^{\circ} / 90^{\circ}\right]_{2 s}$ square plate $(a / h=15)$.

\subsection{Plates with FFFF boundary condition}

In our last example, we consider a laminated composite plate with four free edges (FFFF). This case of boundary conditions has received little attention in the literature, with the noteworthy exception of Araújo dosSantos et al. [44]. They determined the natural frequencies of antisymmetric angle-ply, symmetric cross-ply, and unsymmetric cross-ply FFFF plates with different slenderness ratios $a / h$, using the Ritz method based on classical plate theory (CPT) and higher-order shear deformation theory (HSDT), and the finite element method based on the HSDT (F-HSDT). Their results for the first five natural frequencies are listed in Tables 7, 8, and 9, where the results obtained by the present method are also shown for comparison. In the considered examples, the plane dimensions of all plates are taken as $a=0.2 \mathrm{~m}$ and $b=0.1 \mathrm{~m}$; all layers are considered of the same thickness, and their mechanical properties are those given in [44]. A very good agreement can be observed between the present and former methods. As an example, the first five mode shapes for the antisymmetric angle-ply plate with $a / h=10$ are shown in figure 8 . 
Table 7

First five natural frequencies $(\mathrm{Hz})$ of an antisymmetric angle-ply $\left[-45^{\circ} / 45^{\circ} /-45^{\circ} / 45^{\circ} /-45^{\circ} / 45^{\circ}\right]$ plate with FFFF edges and various slenderness ratios

\begin{tabular}{|c|c|c|c|c|c|c|}
\hline \multirow{2}{*}{$\begin{array}{l}\text { Slenderness } \\
\text { ratio }(a / h)\end{array}$} & \multirow{2}{*}{ Method } & \multicolumn{5}{|c|}{ Mode number } \\
\hline & & 1 & 2 & 3 & 4 & 5 \\
\hline \multirow{4}{*}{5} & CPT [44] & 3188.9 & 5698.8 & 7102.0 & 7816.4 & 9150.8 \\
\hline & HSDT [44] & 2878.9 & 4530.2 & 5687.1 & 6522.9 & 7722.1 \\
\hline & F-HSDT [44] & 2853.8 & 4451.2 & 5638.4 & 6447.5 & 7699.2 \\
\hline & Present & 2863.0 & 4553.0 & 5689.2 & 6559.6 & 7714.0 \\
\hline \multirow{4}{*}{10} & CPT [44] & 1689.8 & 3813.5 & 5081.9 & 5762.3 & 7619.1 \\
\hline & HSDT [44] & 1620.7 & 3080.6 & 4306.2 & 5719.2 & 5896.4 \\
\hline & F-HSDT [44] & 1612.7 & 3056.8 & 4278.7 & 5686.8 & 5831.0 \\
\hline & Present & 1617.6 & 3057.3 & 4279.0 & 5709.4 & 5832.4 \\
\hline \multirow{4}{*}{20} & CPT [44] & 858.1 & 1938.7 & 2646.4 & 4027.7 & 4046.1 \\
\hline & HSDT [44] & 844.3 & 1764.3 & 2456.9 & 3566.5 & 3805.8 \\
\hline & F-HSDT [44] & 841.9 & 1758.8 & 2448.0 & 3549.6 & 3797.9 \\
\hline & Present & 843.7 & 1758.8 & 2450.2 & 3549.7 & 3797.6 \\
\hline \multirow{4}{*}{30} & CPT [44] & 573.8 & 1296.5 & 1776.2 & 2710.3 & 2714.4 \\
\hline & HSDT [44] & 568.2 & 1222.2 & 1697.3 & 2513.2 & 2632.8 \\
\hline & F-HSDT [44] & 567.1 & 1219.9 & 1693.1 & 2505.1 & 2629.7 \\
\hline & Present & 568.0 & 1219.9 & 1694.6 & 2505.9 & 2629.5 \\
\hline \multirow{4}{*}{40} & CPT [44] & 430.8 & 973.5 & 1335.3 & 2036.1 & 2043.6 \\
\hline & HSDT [44] & 427.8 & 932.9 & 1292.9 & 1932.4 & 2003.9 \\
\hline & F-HSDT [44] & 427.1 & 931.5 & 1290.4 & 1926.7 & 2002.1 \\
\hline & Present & 427.7 & 931.6 & 1291.4 & 1926.8 & 2002.2 \\
\hline \multirow{4}{*}{50} & CPT [44] & 344.8 & 779.2 & 1069.4 & 1630.2 & 1637.8 \\
\hline & HSDT [44] & 343.0 & 753.8 & 1043.3 & 1567.4 & 1615.2 \\
\hline & F-HSDT [44] & 342.5 & 752.8 & 1041.4 & 1562.8 & 1613.9 \\
\hline & Present & 342.9 & 752.9 & 1042.1 & 1562.9 & 1614.0 \\
\hline \multirow{4}{*}{100} & CPT [44] & 172.5 & 389.9 & 535.5 & 815.9 & 820.9 \\
\hline & HSDT [44] & 172.1 & 384.2 & 530.1 & 803.5 & 817.1 \\
\hline & F-HSDT [44] & 171.9 & 383.5 & 528.9 & 800.6 & 816.5 \\
\hline & Present & 172.1 & 383.5 & 529.1 & 800.6 & 816.6 \\
\hline
\end{tabular}

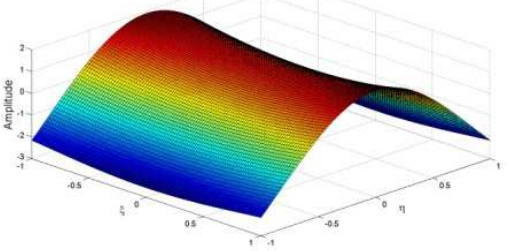

(a) First mode

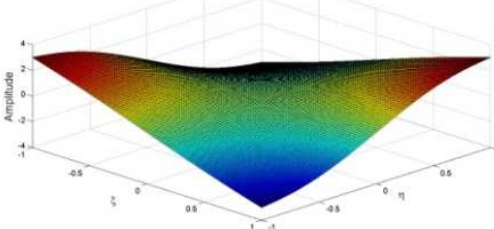

(b) Second mode

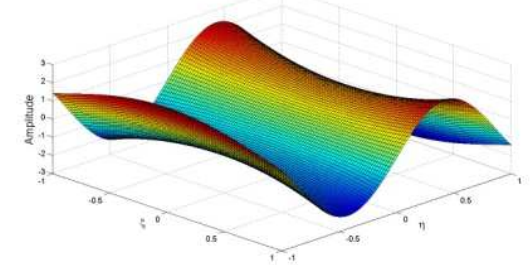

(c) Third mode

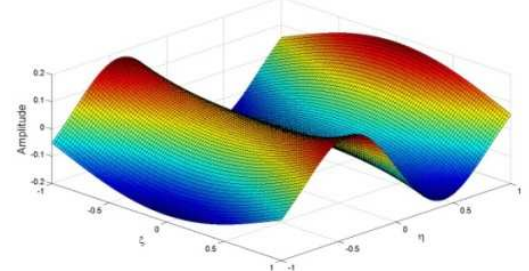

(d) Fourth mode

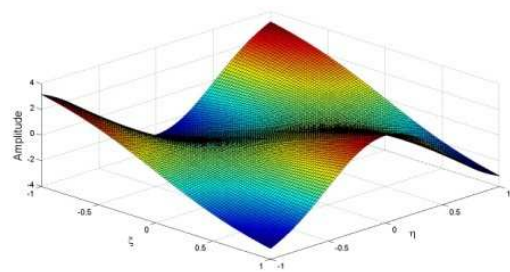

(e) Fifth mode

Fig. 8. First five mode shapes of a $\left[-45^{\circ} / 45^{\circ} /-45^{\circ} / 45^{\circ} /-45^{\circ} / 45^{\circ}\right]$ plate with FFFF edges $(a / h=10)$. 
Table 8

First five natural frequencies $(\mathrm{Hz})$ of a symmetric cross-ply $\left[0^{\circ} / 90^{\circ} / 0^{\circ}\right]$ plate with $\mathrm{FFFF}$ edges and various slenderness ratios

\begin{tabular}{|c|c|c|c|c|c|c|}
\hline \multirow{2}{*}{$\begin{array}{l}\text { Slenderness } \\
\text { ratio }(a / h)\end{array}$} & \multirow{2}{*}{ Method } & \multicolumn{5}{|c|}{ Mode number } \\
\hline & & 1 & 2 & 3 & 4 & 5 \\
\hline \multirow{4}{*}{5} & CPT [44] & 3422.9 & 7265.9 & 8591.3 & 9586.5 & 10282.9 \\
\hline & HSDT [44] & 2759.7 & 5252.0 & 6454.2 & 7634.0 & 8421.8 \\
\hline & F-HSDT [44] & 2760.4 & 5243.3 & 6439.1 & 7599.2 & 8387.9 \\
\hline & Present & 2766.3 & 5286.2 & 6465.0 & 7688.1 & 8432.2 \\
\hline \multirow{4}{*}{10} & CPT [44] & 1816.1 & 3836.7 & 5061.3 & 5245.0 & 6104.5 \\
\hline & HSDT [44] & 1637.9 & 3403.6 & 4404.1 & 4792.8 & 5494.8 \\
\hline & F-HSDT [44] & 1638.3 & 3407.2 & 4407.8 & 4785.8 & 5488.6 \\
\hline & Present & 1637.8 & 3404.2 & 4404.2 & 4795.0 & 5490.1 \\
\hline \multirow{4}{*}{20} & CPT [44] & 922.7 & 1945.9 & 2663.8 & 2689.3 & 3220.1 \\
\hline & HSDT [44] & 880.7 & 1880.0 & 2528.0 & 2618.5 & 3087.5 \\
\hline & F-HSDT [44] & 880.3 & 1880.5 & 2526.5 & 2618.8 & 3087.3 \\
\hline & Present & 880.6 & 1880.8 & 2527.3 & 2618.9 & 3087.9 \\
\hline \multirow{4}{*}{30} & CPT [44] & 617.0 & 1300.8 & 1793.9 & 1801.5 & 2169.5 \\
\hline & HSDT [44] & 599.7 & 1280.4 & 1744.7 & 1779.6 & 2119.4 \\
\hline & F-HSDT [44] & 598.9 & 1280.3 & 1741.9 & 1780.4 & 2119.0 \\
\hline & Present & 599.3 & 1280.5 & 1742.0 & 1781.6 & 2119.3 \\
\hline \multirow{4}{*}{40} & CPT [44] & 463.2 & 976.5 & 1350.2 & 1353.4 & 1633.2 \\
\hline & HSDT [44] & 454.2 & 967.8 & 1327.0 & 1344.1 & 1608.8 \\
\hline & F-HSDT [44] & 453.3 & 967.6 & 1324.0 & 1344.9 & 1607.9 \\
\hline & Present & 453.5 & 967.6 & 1324.3 & 1344.6 & 1608.2 \\
\hline \multirow{4}{*}{50} & CPT [44] & 370.8 & 781.5 & 1082.0 & 1083.6 & 1308.8 \\
\hline & HSDT [44] & 365.5 & 777.0 & 1069.1 & 1078.8 & 1295.1 \\
\hline & F-HSDT [44] & 364.6 & 776.8 & 1066.5 & 1079.5 & 1294.0 \\
\hline & Present & 364.8 & 776.9 & 1066.7 & 1078.6 & 1294.8 \\
\hline \multirow{4}{*}{100} & CPT [44] & 185.5 & 391.0 & 542.2 & 542.3 & 655.9 \\
\hline & HSDT [44] & 184.6 & 390.4 & 540.3 & 541.8 & 653.9 \\
\hline & F-HSDT [44] & 184.0 & 390.3 & 539.0 & 542.2 & 653.1 \\
\hline & Present & 184.4 & 390.4 & 540.7 & 541.8 & 653.1 \\
\hline
\end{tabular}

Table 9

First five natural frequencies $(\mathrm{Hz})$ of an unsymmetric cross-ply $\left[0^{\circ} / 90^{\circ}\right]$ plate with FFFF edges and various slenderness ratios

\begin{tabular}{|c|c|c|c|c|c|c|}
\hline \multirow{2}{*}{$\begin{array}{l}\text { Slenderness } \\
\text { ratio }(a / h)\end{array}$} & \multirow{2}{*}{ Method } & \multicolumn{5}{|c|}{ Mode number } \\
\hline & & 1 & 2 & 3 & 4 & 5 \\
\hline \multirow{4}{*}{5} & CPT [44] & 3384.0 & 3590.6 & 6085.0 & 8289.0 & 8299.8 \\
\hline & HSDT [44] & 2753.3 & 3294.7 & 5413.5 & 6860.3 & 7123.8 \\
\hline & F-HSDT [44] & 2742.6 & 3196.1 & 5355.3 & 6632.0 & 6829.6 \\
\hline & Present & 2743.4 & 3284.2 & 5368.5 & 6942.2 & 7001.8 \\
\hline \multirow{4}{*}{10} & CPT [44] & 1803.7 & 1968.6 & 4003.7 & 5244.6 & 6360.8 \\
\hline & HSDT [44] & 1636.1 & 1905.4 & 3561.6 & 4734.6 & 5890.2 \\
\hline & F-HSDT [44] & 1633.0 & 1886.3 & 3540.5 & 4596.4 & 5867.7 \\
\hline & Present & 1633.3 & 1904.8 & 3543.6 & 4702.1 & 5878.2 \\
\hline \multirow{4}{*}{20} & CPT [44] & 917.6 & 1009.4 & 2104.2 & 2757.7 & 3403.0 \\
\hline & HSDT [44] & 877.9 & 1000.0 & 1999.5 & 2668.4 & 3314.9 \\
\hline & F-HSDT [44] & 877.0 & 997.0 & 1992.9 & 2643.5 & 3313.3 \\
\hline & Present & 877.0 & 1000.0 & 1994.2 & 2664.0 & 3312.1 \\
\hline \multirow{4}{*}{30} & CPT [44] & 613.7 & 676.1 & 1414.9 & 1856.5 & 2299.1 \\
\hline & HSDT [44] & 597.1 & 673.3 & 1374.3 & 1828.0 & 2270.6 \\
\hline & F-HSDT [44] & 596.4 & 672.2 & 1369.8 & 1819.9 & 2271.1 \\
\hline & Present & 596.5 & 673.3 & 1370.2 & 1826.6 & 2271.5 \\
\hline \multirow{4}{*}{40} & CPT [44] & 460.8 & 508.0 & 1064.3 & 1397.2 & 1732.5 \\
\hline & HSDT [44] & 452.0 & 506.7 & 1044.2 & 1384.8 & 1720.1 \\
\hline & F-HSDT [44] & 451.3 & 506.2 & 1040.6 & 1381.2 & 1720.9 \\
\hline & Present & 451.3 & 506.7 & 1040.9 & 1384.2 & 1720.3 \\
\hline \multirow{4}{*}{50} & CPT [44] & 368.9 & 406.7 & 852.6 & 1119.6 & 1389.1 \\
\hline & HSDT [44] & 363.1 & 406.0 & 839.9 & 1112.2 & 1381.7 \\
\hline & F-HSDT [44] & 362.9 & 405.7 & 838.2 & 1111.2 & 1383.5 \\
\hline & Present & 362.8 & 406.0 & 838.5 & 1112.8 & 1381.7 \\
\hline
\end{tabular}

DOI: https://doi.org/10.1016/j.compstruc.2016.07.007 


\begin{tabular}{llllll} 
CPT [44] & 184.6 & 203.6 & 427.1 & 561.0 & 696.6 \\
\hline HSDT [44] & 183.7 & 203.5 & 425.3 & 560.2 & 695.8 \\
\hline F-HSDT [44] & 183.1 & 203.4 & 423.9 & 559.9 & 696.3 \\
\hline Present & 183.3 & 203.5 & 425.5 & 560.1 & 695.6 \\
\hline
\end{tabular}

\section{Conclusions}

A new solution method has been presented for the free vibration analysis of rectangular laminated composite plates. A strength of the method is the possibility of analysing plates with general stacking sequences, including unsymmetric laminates with elastic couplings. Furthermore, any boundary condition corresponding to clamped, simply supported, free - as well as elastically supported - edges can be considered, thanks to the introduction of fictitious elastic restraints on the plate's four edges.

Shear deformability, which may be relevant for laminated composite plates, is taken into account. However, for the sake of simplicity, we adopt first-order (and not higher-order) shear deformation theory. Accordingly, the plate's displacement field is expressed in terms of the mid-plane displacements and rotations. The governing differential equations and boundary conditions have been derived by using Hamilton's principle. To solve specific problems, the generalised displacements are expanded as series of Legendre polynomials. Then, an energy functional - definedas the sum of strain and kinetic energies of the plate - is extremised subjected to the boundary conditions. The latter are imposed via the method of Lagrange multipliers. As a result, a classical eigenvalue problem in matrix form is obtained, out of which the natural frequencies and mode shapes can be calculated. It is worth emphasising, as an advantage of the method, that the properties of the Legendre polynomials enable significant simplification of the expression of the energy functional. Furthermore, the method of Lagrange multipliers allows us to use displacement functions that do not have to satisfy a priori the boundary conditions of the problem. It should be mentioned that in order to overcome some difficulties in calculating the natural frequencies and corresponding mode shapes, a new numerical strategy has also been proposed.

The proposed method has been applied to several example cases. The obtained results have been compared with those available in the literature and good agreement has been obtained. Only for very thick plates, slight differences in the natural frequencies have emerged from the comparison with more accurate theories accounting for the transverse normal and shear strain and stress. Better results could probably have been obtained by considering higher-order shear deformation theory. This possible development of the method will be the subject of future studies. Finally, a parametric study has been conducted to evaluate the effects of boundary conditions, stacking sequence, material anisotropy, spanto-thickness, and aspect ratios on the vibrational characteristics of the plate.

\section{Appendix A}

According to the first-order shear deformation theory, the constitutive equations of the laminated plate can be expressed as follows $[1,10]$ :

$$
\begin{aligned}
& \left\{\begin{array}{l}
N_{x} \\
N_{y} \\
N_{x y} \\
M_{x} \\
M_{y} \\
M_{x y}
\end{array}\right\}=\left[\begin{array}{llllll}
A_{11} & A_{12} & A_{16} & B_{11} & B_{12} & B_{16} \\
A_{12} & A_{22} & A_{26} & B_{12} & B_{22} & B_{26} \\
A_{16} & A_{26} & A_{66} & B_{16} & B_{26} & B_{66} \\
B_{11} & B_{12} & B_{16} & D_{11} & D_{12} & D_{16} \\
B_{12} & B_{22} & B_{26} & D_{12} & D_{22} & D_{26} \\
B_{16} & B_{26} & B_{66} & D_{16} & D_{26} & D_{66}
\end{array}\right]\left\{\begin{array}{l}
\varepsilon_{x}^{0} \\
\varepsilon_{y}^{0} \\
\gamma_{x y}^{0} \\
\kappa_{x} \\
\kappa_{y} \\
\kappa_{x y}
\end{array}\right\}, \\
& \left\{\begin{array}{l}
Q_{y z} \\
Q_{x z}
\end{array}\right\}=\left[\begin{array}{ll}
A_{44} & A_{45} \\
A_{45} & A_{55}
\end{array}\right]\left\{\begin{array}{l}
\gamma_{y z} \\
\gamma_{x z}
\end{array}\right\},
\end{aligned}
$$

where $A_{i j}, B_{i j}$, and $D_{i j}(i, j \in\{1,2,6\})$ are the extension, bending-extension coupling, and bending stiffnesses, respectively, and $A_{i j}(i, j \in\{4,5\})$ are the transverse shear stiffnesses. These quantities can be computed as:

$$
\begin{aligned}
A_{i j} & =\sum_{k=1}^{N L} \bar{Q}_{i j}{ }^{(k)}\left(z_{k}-z_{k-1}\right), \quad B_{i j}=\frac{1}{2} \sum_{k=1}^{N L} \bar{Q}_{i j}^{(k)}\left(z_{k}{ }^{2}-z_{k-1}{ }^{2}\right), \quad \text { and } D_{i j}=\frac{1}{3} \sum_{k=1}^{N L} \bar{Q}_{i j}^{(k)}\left(z_{k}{ }^{3}-z_{k-1}{ }^{3}\right), \quad \text { with } i, j \in\{1,2,6\} ; \\
A_{i j} & =k_{s} \sum_{k=1}^{N L} \bar{Q}_{i j}^{(k)}\left(z_{k}-z_{k-1}\right), \quad \text { with } i, j \in\{4,5\} ;
\end{aligned}
$$

where $\bar{Q}_{i j}{ }^{(k)}$ are the transformed stiffness of the $k^{\text {th }}$ layer as expressed in [1]. Besides, $k_{s}$ is the shear correction factor,

$N L$ is the total number of layers in the laminate, $z_{k}$ and $z_{k-1}$ are the distances of the top and bottom surfaces of the $k^{\text {th }}$ layer from the plate's mid-plane, respectively.

The strain energy of the plate can be calculated as:

$$
U_{P}=\frac{1}{2} \int_{0}^{a} \int_{0}^{b}\left(N_{x} \varepsilon_{x}^{0}+N_{y} \varepsilon_{y}^{0}+N_{x y} \gamma_{x y}^{0}+M_{x} \kappa_{x}+M_{y} \kappa_{y}+M_{x y} \kappa_{x y}+Q_{y z} \gamma_{y z}+Q_{x z} \gamma_{x z}\right) d y d x
$$


where $\varepsilon_{x}^{0}, \varepsilon_{y}^{0}$, and $\gamma_{x y}^{0}$ are the mid-plane strains, $\kappa_{x}$ and $\kappa_{y}$ are the bending curvatures, $\kappa_{x y}$ is the twisting curvature, $\gamma_{y z}$ and $\gamma_{x z}$ are the shear strains. Moreover, the strain energy stored in the elastic edge supports is:

$$
\begin{aligned}
U_{E}= & \frac{1}{2} \int_{0}^{b}\left[k_{u}^{x=0} u^{2}(0, y)+k_{v}^{x=0} v^{2}(0, y)+k_{w}^{x=0} w^{2}(0, y)+k_{\phi}^{x=0} \phi^{2}(0, y)+k_{\psi}^{x=0} \psi^{2}(0, y)\right] d y \\
& +\frac{1}{2} \int_{0}^{b}\left[k_{u}^{x=a} u^{2}(a, y)+k_{v}^{x=a} v^{2}(a, y)+k_{w}^{x=a} w^{2}(a, y)+k_{\phi}^{x=a} \phi^{2}(a, y)+k_{\psi}^{x=a} \psi^{2}(a, y)\right] d y \\
& +\frac{1}{2} \int_{0}^{a}\left[k_{u}^{y=0} u^{2}(x, 0)+k_{v}^{y=b} v^{2}(x, 0)+k_{w}^{y=b} w^{2}(x, 0)+k_{\phi}^{y=b} \phi^{2}(x, 0)+k_{\psi}^{y=0} \psi^{2}(x, 0)\right] d x \\
& +\frac{1}{2} \int_{0}^{a}\left[k_{u}^{y=b} u^{2}(x, b)+k_{v}^{y=b} v^{2}(x, b)+k_{w}^{y=b} w^{2}(x, b)+k_{\phi}^{y=b} \phi^{2}(x, b)+k_{\psi}^{y=b} \psi^{2}(x, b)\right] d x .
\end{aligned}
$$

The relations for the above stated strains and curvatures versus the displacement field can be written as follows [10]: $\varepsilon_{x}^{0}=u_{, x}, \quad \varepsilon_{y}^{0}=v_{, y}, \quad \gamma_{x y}^{0}=u_{, y}+v_{, x}$,

$\kappa_{x}=\phi_{, x}, \quad \kappa_{y}=\psi_{, y}, \quad \kappa_{x y}=\phi_{, y}+\psi_{, x}$,

$\gamma_{x z}=\phi+w_{, x}, \quad \gamma_{y z}=\psi+w_{, y}$,

Based on first-order shear deformation theory, the kinetic energy of the laminated plate can be expressed as:

$T=\frac{1}{2} \int_{0}^{a} \int_{0}^{b}\left[I_{0}\left(u_{, t}^{2}+v_{, t}^{2}+w_{, t}^{2}\right)+2 I_{1}\left(u_{, t} \phi_{, t}+v_{, t} \psi_{, t}\right)+I_{2}\left(\phi_{, t}^{2}+\psi_{, t}^{2}\right)\right] d y d x$,

where:

$\left(I_{0}, I_{1}, I_{2}\right)=\int_{-h / 2}^{h / 2} \rho(z)\left(1, z, z^{2}\right) d z$

are the mass moments of inertia and $\rho(z)$ is the density of the plate (commonly, the density is assumed constant within each constituent layer). It is noted that, if the centre of mass is placed on the plate's mid-plane, then $I_{1}=0$. This happens, for instance, for homogeneous and symmetrically laminated plates [10].

\section{Appendix B}

By using the same procedure adopted to obtain Eq. (11), the following expressions are determined for the remaining 19 boundary conditions. Along the edge $\xi=-1$ :

$$
\begin{aligned}
\mathbf{C}_{2 j}: & \frac{2}{a} A_{16} \Re_{i m}(-1)^{m} U_{i j}+\frac{2}{b} A_{26} \Re_{l j}(-1)^{i} V_{i l}+A_{66}\left\{\frac{2}{b} \Re_{l j}(-1)^{i} U_{i l}+\frac{2}{a} \Re_{i m}(-1)^{m} V_{i j}\right\}+\frac{2}{a} B_{16} \Re_{i m}(-1)^{m} \Phi_{i j} \\
& +\frac{2}{b} B_{26} \Re_{l j}(-1)^{i} \Psi_{i l}+B_{66}\left\{\frac{2}{b} \Re_{l j}(-1)^{i} \Phi_{i l}+\frac{2}{a} \Re_{i m}(-1)^{m} \Psi_{i j}\right\}-k_{v}^{x=0}(-1)^{i} V_{i j}=0, \quad j=0,1, \ldots, J, \\
\mathbf{C}_{3 j}: & \frac{2}{a} B_{11} \Re_{i m}(-1)^{m} U_{i j}+\frac{2}{b} B_{12} \Re_{l j}(-1)^{i} V_{i l}+B_{16}\left\{\frac{2}{b} \Re_{l j}(-1)^{i} U_{i l}+\frac{2}{a} \Re_{i m}(-1)^{m} V_{i j}\right\}+\frac{2}{a} D_{11} \Re_{i m}(-1)^{m} \Phi_{i j} \\
& +\frac{2}{b} D_{12} \Re_{l j}(-1)^{i} \Psi_{i l}+D_{16}\left\{\frac{2}{b} \Re_{l j}(-1)^{i} \Phi_{i l}+\frac{2}{a} \Re_{i m}(-1)^{m} \Psi_{i j}\right\}-k_{\psi_{x}}^{x=0}(-1)^{i} \Phi_{i j}=0, \quad j=0,1, \ldots, J, \\
\mathbf{C}_{4 j}: & \frac{2}{a} B_{16} \Re_{i m}(-1)^{m} U_{i j}+\frac{2}{b} B_{26} \Re_{l j}(-1)^{i} V_{i l}+B_{66}\left\{\frac{2}{b} \Re_{l j}(-1)^{i} U_{i l}+\frac{2}{a} \Re_{i m}(-1)^{m} V_{i j}\right\}+\frac{2}{a} D_{16} \Re_{i m}(-1)^{m} \Phi_{i j} \\
& +\frac{2}{b} D_{26} \Re_{l j}(-1)^{i} \Psi_{i l}+D_{66}\left\{\frac{2}{b} \Re_{l j}(-1)^{i} \Phi_{i l}+\frac{2}{a} \Re_{i m}(-1)^{m} \Psi_{i j}\right\}-k_{\psi y}^{x=0}(-1)^{i} \Psi_{i j}=0, \quad j=0,1, \ldots, J, \\
\mathbf{C}_{5 j}: & A_{45}(-1)^{i} \Psi_{i j}+\frac{2}{b} A_{45} \Re_{l j}(-1)^{i} W_{i l}+A_{55}(-1)^{i} \Phi_{i j}+\frac{2}{a} A_{55} \Re_{i m}(-1)^{m} W_{i j}-k_{w}^{x=0}(-1)^{i} W_{i j}=0, \quad j=0,1, \ldots, J ;
\end{aligned}
$$

along the edge $\xi=1$ :

$$
\begin{aligned}
\mathbf{C}_{6 j}: & \frac{2}{a} A_{11} \Re_{i m}(+1)^{m} U_{i j}+\frac{2}{b} A_{12} \Re_{l j}(+1)^{i} V_{i l}+A_{16}\left\{\frac{2}{b} \Re_{l j}(+1)^{i} U_{i l}+\frac{2}{a} \Re_{i m}(+1)^{m} V_{i j}\right\}+\frac{2}{a} B_{11} \Re_{i m}(+1)^{m} \Phi_{i j} \\
& +\frac{2}{b} B_{12} \Re_{l j}(+1)^{i} \Psi_{i l}+B_{16}\left\{\frac{2}{b} \Re_{l j}(+1)^{i} \Phi_{i l}+\frac{2}{a} \Re_{i m}(+1)^{m} \Psi_{i j}\right\}+k_{u}^{x=a}(+1)^{i} U_{i j}=0, \quad j=0,1, \ldots, J, \\
\mathbf{C}_{7 j}: & \frac{2}{a} A_{16} \Re_{i m}(+1)^{m} U_{i j}+\frac{2}{b} A_{26} \Re_{l j}(+1)^{i} V_{i l}+A_{66}\left\{\frac{2}{b} \Re_{l j}(+1)^{i} U_{i l}+\frac{2}{a} \Re_{i m}(+1)^{m} V_{i j}\right\}+\frac{2}{a} B_{16} \Re_{i m}(+1)^{m} \Phi_{i j} \\
& +\frac{2}{b} B_{26} \Re_{l j}(+1)^{i} \Psi_{i l}+B_{66}\left\{\frac{2}{b} \Re_{l j}(+1)^{i} \Phi_{i l}+\frac{2}{a} \Re_{i m}(+1)^{m} \Psi_{i j}\right\}+k_{v}^{x=a}(+1)^{i} V_{i j}=0, \quad j=0,1, \ldots, J, \\
\mathbf{C}_{8 j}: & \frac{2}{a} B_{11} \Re_{i m}(+1)^{m} U_{i j}+\frac{2}{b} B_{12} \Re_{l j}(+1)^{i} V_{i l}+B_{16}\left\{\frac{2}{b} \Re_{l j}(+1)^{i} U_{i l}+\frac{2}{a} \Re_{i m}(+1)^{m} V_{i j}\right\}+\frac{2}{a} D_{11} \Re_{i m}(+1)^{m} \Phi_{i j} \\
& +\frac{2}{b} D_{12} \Re_{l j}(+1)^{i} \Psi_{i l}+D_{16}\left\{\frac{2}{b} \Re_{l j}(+1)^{i} \Phi_{i l}+\frac{2}{a} \Re_{i m}(+1)^{m} \Psi_{i j}\right\}+k_{\psi_{x}}^{x=a}(+1)^{i} \Phi_{i j}=0, \quad j=0,1, \ldots, J, \\
\mathbf{C}_{9 j}: & \frac{2}{a} B_{16} \Re_{i m}(+1)^{m} U_{i j}+\frac{2}{b} B_{26} \Re_{l j}(+1)^{i} V_{i l}+B_{66}\left\{\frac{2}{b} \Re_{l j}(+1)^{i} U_{i l}+\frac{2}{a} \Re_{i m}(+1)^{m} V_{i j}\right\}+\frac{2}{a} D_{16} \Re_{i m}(+1)^{m} \Phi_{i j} \\
& +\frac{2}{b} D_{26} \Re_{l j}(+1)^{i} \Psi_{i l}+D_{66}\left\{\frac{2}{b} \Re_{l j}(+1)^{i} \Phi_{i l}+\frac{2}{a} \Re_{i m}(+1)^{m} \Psi_{i j}\right\}+k_{\psi_{y}}^{x=a}(+1)^{i} \Psi_{i j}=0, \quad j=0,1, \ldots, J,
\end{aligned}
$$

DOI: https://doi.org/10.1016/j.compstruc.2016.07.007 
$\mathbf{C}_{10 j}: \quad A_{45}(+1)^{i} \Psi_{i j}+\frac{2}{b} A_{45} \Re_{l j}(+1)^{i} W_{i l}+A_{55}(+1)^{i} \Phi_{i j}+\frac{2}{a} A_{55} \Re_{i m}(+1)^{m} W_{i j}+k_{w}^{x=a}(+1)^{i} W_{i j}=0, \quad j=0,1, \ldots, J ;$

along the edge $\eta=-1$ :

$$
\begin{aligned}
\mathbf{C}_{11 i}: & \frac{2}{a} A_{12} \Re_{k i}(-1)^{j} U_{k j}+\frac{2}{b} A_{22} \Re_{j n}(-1)^{n} V_{i j}+A_{26}\left\{\frac{2}{b} \Re_{j n}(-1)^{n} U_{i j}+\frac{2}{a} \Re_{k i}(-1)^{j} V_{k j}\right\}+\frac{2}{a} B_{12} \Re_{k i}(-1)^{j} \Phi_{k j} \\
& +\frac{2}{b} B_{22} \Re_{j n}(-1)^{n} \Psi_{i j}+B_{26}\left\{\frac{2}{b} \Re_{j n}(-1)^{n} \Phi_{i j}+\frac{2}{a} \Re_{k i}(-1)^{j} \Psi_{k j}\right\}-k_{v}^{y=0}(-1)^{j} V_{i j}=0, \quad i=0,1, \ldots, I, \\
\mathbf{C}_{12 i}: & \frac{2}{a} A_{16} \Re_{k i}(-1)^{j} U_{k j}+\frac{2}{b} A_{26} \Re_{j n}(-1)^{n} V_{i j}+A_{66}\left\{\frac{2}{b} \Re_{j n}(-1)^{n} U_{i j}+\frac{2}{a} \Re_{k i}(-1)^{j} V_{k j}\right\}+\frac{2}{a} B_{16} \Re_{k i}(-1)^{j} \Phi_{k j} \\
& +\frac{2}{b} B_{26} \Re_{j n}(-1)^{n} \Psi_{i j}+B_{66}\left\{\frac{2}{b} \Re_{j n}(-1)^{n} \Phi_{i j}+\frac{2}{a} \Re_{k i}(-1)^{j} \Psi_{k j}\right\}-k_{u}^{y=0}(-1)^{j} U_{i j}=0, \quad i=0,1, \ldots, I, \\
\mathbf{C}_{13 i}: & \frac{2}{a} B_{12} \Re_{k i}(-1)^{j} U_{k j}+\frac{2}{b} B_{22} \Re_{j n}(-1)^{n} V_{i j}+B_{26}\left\{\frac{2}{b} \Re_{j n}(-1)^{n} U_{i j}+\frac{2}{a} \Re_{k i}(-1)^{j} V_{k j}\right\}+\frac{2}{a} D_{12} \Re_{k i}(-1)^{j} \Phi_{k j} \\
& +\frac{2}{b} D_{22} \Re_{j n}(-1)^{n} \Psi_{i j}+D_{26}\left\{\frac{2}{b} \Re_{j n}(-1)^{n} \Phi_{i j}+\frac{2}{a} \Re_{k i}(-1)^{j} \Psi_{k j}\right\}-k_{\psi_{y}}^{y=0}(-1)^{j} \Psi_{i j}=0, \quad i=0,1, \ldots, I, \\
\mathbf{C}_{14 i}: & \frac{2}{a} B_{16} \Re_{k i}(-1)^{j} U_{k j}+\frac{2}{b} B_{26} \Re_{j n}(-1)^{n} V_{i j}+B_{66}\left\{\frac{2}{b} \Re_{j n}(-1)^{n} U_{i j}+\frac{2}{a} \Re_{k i}(-1)^{j} V_{k j}\right\}+\frac{2}{a} D_{16} \Re_{k i}(-1)^{j} \Phi_{k j} \\
& +\frac{2}{b} D_{26} \Re_{j n}(-1)^{n} \Psi_{i j}+D_{66}\left\{\frac{2}{b} \Re_{j n}(-1)^{n} \Phi_{i j}+\frac{2}{a} \Re_{k i}(-1)^{j} \Psi_{k j}\right\}-k_{\psi_{x}}^{y=0}(-1)^{j} \Phi_{i j}=0, \quad i=0,1, \ldots, I, \\
\mathbf{C}_{15 i}: & A_{44}(-1)^{j} \Psi_{i j}+\frac{2}{b} A_{44} \Re_{j n}(-1)^{n} W_{i j}+A_{45}(-1)^{j} \Phi_{i j}+\frac{2}{a} A_{45} \Re_{k i}(-1)^{j} W_{k j}-k_{w}^{y=0}(-1)^{j} W_{i j}=0, \quad i=0,1, \ldots, I ;
\end{aligned}
$$

along the edge $\eta=1$

$$
\begin{aligned}
\mathbf{C}_{16 i}: & \frac{2}{a} A_{12} \Re_{k i}(+1)^{j} U_{k j}+\frac{2}{b} A_{22} \Re_{j n}(+1)^{n} V_{i j}+A_{26}\left\{\frac{2}{b} \Re_{j n}(+1)^{n} U_{i j}+\frac{2}{a} \Re_{k i}(+1)^{j} V_{k j}\right\}+\frac{2}{a} B_{12} \Re_{k i}(+1)^{j} \Phi_{k j} \\
& +\frac{2}{b} B_{22} \Re_{j n}(+1)^{n} \Psi_{i j}+B_{26}\left\{\frac{2}{b} \Re_{j n}(+1)^{n} \Phi_{i j}+\frac{2}{a} \Re_{k i}(+1)^{j} \Psi_{k j}\right\}+k_{v}^{y=b}(+1)^{j} V_{i j}=0, \quad i=0,1, \ldots, I, \\
\mathbf{C}_{17 i}: & \frac{2}{a} A_{16} \Re_{k i}(+1)^{j} U_{k j}+\frac{2}{b} A_{26} \Re_{j n}(+1)^{n} V_{i j}+A_{66}\left\{\frac{2}{b} \Re_{j n}(+1)^{n} U_{i j}+\frac{2}{a} \Re_{k i}(+1)^{j} V_{k j}\right\}+\frac{2}{a} B_{16} \Re_{k i}(+1)^{j} \Phi_{k j} \\
& +\frac{2}{b} B_{26} \Re_{j n}(+1)^{n} \Psi_{i j}+B_{66}\left\{\frac{2}{b} \Re_{j n}(+1)^{n} \Phi_{i j}+\frac{2}{a} \Re_{k i}(+1)^{j} \Psi_{k j}\right\}+k_{u}^{y=b}(+1)^{j} U_{i j}=0, \quad i=0,1, \ldots, I, \\
\mathbf{C}_{18 i}: & \frac{2}{a} B_{12} \Re_{k i}(+1)^{j} U_{k j}+\frac{2}{b} B_{22} \Re_{j n}(+1)^{n} V_{i j}+B_{26}\left\{\frac{2}{b} \Re_{j n}(+1)^{n} U_{i j}+\frac{2}{a} \Re_{k i}(+1)^{j} V_{k j}\right\}+\frac{2}{a} D_{12} \Re_{k i}(+1)^{j} \Phi_{k j} \\
& +\frac{2}{b} D_{22} \Re_{j n}(+1)^{n} \Psi_{i j}+D_{26}\left\{\frac{2}{b} \Re_{j n}(+1)^{n} \Phi_{i j}+\frac{2}{a} \Re_{k i}(+1)^{j} \Psi_{k j}\right\}+k_{\psi y}^{y=b}(+1)^{j} \Psi_{i j}=0, \quad i=0,1, \ldots, I, \\
\mathbf{C}_{19 i}: & \frac{2}{a} B_{16} \Re_{k i}(+1)^{j} U_{k j}+\frac{2}{b} B_{26} \Re_{j n}(+1)^{n} V_{i j}+B_{66}\left\{\frac{2}{b} \Re_{j n}(+1)^{n} U_{i j}+\frac{2}{a} \Re_{k i}(+1)^{j} V_{k j}\right\}+\frac{2}{a} D_{16} \Re_{k i}(+1)^{j} \Phi_{k j} \\
& +\frac{2}{b} D_{26} \Re_{j n}(+1)^{n} \Psi_{i j}+D_{66}\left\{\frac{2}{b} \Re_{j n}(+1)^{n} \Phi_{i j}+\frac{2}{a} \Re_{k i}(+1)^{j} \Psi_{k j}\right\}+k_{\psi_{x}}^{y=b}(+1)^{j} \Phi_{i j}=0, \quad i=0,1, \ldots, I, \\
\mathbf{C}_{20 i}: & A_{44}(+1)^{j} \Psi_{i j}+\frac{2}{b} A_{44} \Re_{j n}(+1)^{n} W_{i j}+A_{45}(+1)^{j} \Phi_{i j}+\frac{2}{a} A_{45} \Re_{k i}(+1)^{j} W_{k j}+k_{w}^{y=b}(+1)^{j} W_{i j}=0, \quad i=0,1, \ldots, I .
\end{aligned}
$$

\section{References}

[1] Jones RM. Mechanics of composite materials $-2^{\text {nd }}$ edition. Philadelphia, PA: Taylor \& Francis Inc., 1999.

[2] Sayyad AS, Ghugal YM. On the free vibration analysis of laminated composite and sandwich plates: A review of recent literature with some numerical results. Compos Struct 2015; 129: 177-201.

[3] Lo KH, Christensen RM, Wu EM. A high-order theory of plate deformation, Part I: Homogeneous plates. J Appl Mech 1977; 44(7): 663-668.

[4] Lo KH, Christensen RM, Wu EM. A high-order theory of plate deformation. Part II: Laminated plates. J Appl Mech 1977; 44(4): 669-676.

[5] Kant T. Numerical analysis of thick plates. Comput Method Appl Mech Eng 1982; 31: 1-18.

[6] Kant T, Owen DRJ, Zienkiewicz OC. A refined higher-order C ${ }^{0}$ plate element. Comput Struct 1982; 15(2): 177183.

[7] Batra RC, Vidoli S. Higher order piezoelectric plate theory derived from a three dimensional variational principle. AIAA J 2002; 40: 91-104.

[8] Carrera E, Kroplin B. Zig-zag and interlaminar equilibria effects in large deflection and post-buckling analysis of multilayered plates. Mech Compos Mater Struct 1997; 4: 69-94.

[9] Librescu L, Khdeir AA, Reddy JN. A comprehensive analysis of the state of stress of elastic anisotropic flat plates using refined theories. Acta Mech 1987; 70: 57-81.

[10] Reddy JN. Theory and analysis of elastic plates and shells $-2^{\text {nd }}$ edition. Boca Raton, FL: CRC Press, 2007.

[11] Fiedler L, Lacarbonara W, Vestroni F. A generalized higher-order theory for multi-layered, shear-deformable composite plates. Acta Mech 2010; 209: 85-98.

DOI: https://doi.org/10.1016/j.compstruc.2016.07.007 
[12] Aghababaei R, Reddy JN. Nonlocal third-order shear deformation plate theory with application to bending and vibration of plates. J Sound Vib 2009; 326(1-2): 277-289.

[13] Kant T, Swaminathan K. Free vibration of isotropic, orthotropic, and multilayer plates based on higher order refined theories. J Sound Vib 2001; 241(2): 319-327.

[14] Robaldo A, Carrera E, Benjeddou A. A unified formulation for finite element analysis of piezoelectric adaptive plates. Comput Struct 2006; 84 (22-23): 1494-1505.

[15] Rao MK, Scherbatiuk K, Desai YM, Shah AH. Natural vibrations of laminated and sandwich plates. ASCE J Eng Mech 2004; 130(11): 1268-1278.

[16] Swaminathan K, Patil SS. Higher order refined computational models for the free vibration analysis of antisymmetric angle ply plates. J Reinf Plast Compos 2008; 27(5): 541-553.

[17] Moita JS, Soares CMM, Soares CAM. Buckling and dynamic behaviour of laminated composite structures using a discrete higher-order displacement model. Comput Struct 1999; 73(1-5): 407-423.

[18] Park T, Lee SY, Seo JW, Voyiadjis GZ. Structural dynamic behaviour of skew sandwich plates with laminated composite faces. Composites: Part B 2008; 39(2): 316-326.

[19] Han W, Petyt M. Linear vibration analysis of laminated rectangular plates using the hierarchical finite element method-I. Free vibration analysis. Comput Struct 1996, 61(4): 705-712.

[20] Rodrigues JD, Natarajan S, Ferreira AJM, Carrera E, Cinefra M, Bordas SPA. Analysis of composite plates through cell-based smoothed finite element and 4-noded mixed interpolation of tensorial components techniques. Comput Struct 2014; 135: 83-87.

[21] Carrera E. Theories and finite elements for multilayered plates and shells: a unified compact formulation with numerical assessment and benchmarking. Arch Comput Methods Eng 2003; 10: 215-96.

[22] Lanhe W, Hua L, Daobin W. Vibration analysis of generally laminated composite plates by the moving least squares differential quadrature method. Compos Struct 2005; 68(3): 319-330.

[23] Ferreira AJM, Carrera E, Cinefra M, Viola E, Tornabene F, Fantuzzi N, Zenkour AM. Analysis of thick isotropic and cross-ply laminated plates by generalized differential quadrature method and a unified formulation. Composites: Part B 2014; 58: 544-552.

[24] Ngo-Cong D, Mai-Duy N, Karunasena W, Tran-Cong T. Free vibration analysis of laminated composite plates based on FSDT using one-dimensional IRBFN method. Comput Struct 2011; 89: 1-13.

[25] Chakraverty S. Vibration of plates. Boca Raton, FL: CRC Press, 2008.

[26] Oosterhout GM, van der Hoogt PJM, Spiering RMEJ. Accurate calculation methods for natural frequencies of plates with special attention to the higher modes. J Sound Vib 1995; 183(1): 33-47.

[27] Chakraverty S, Bhat RB, Stiharu I. Recent research on vibration of structures using boundary characteristic orthogonal polynomials in Rayleigh-Ritz method. Shock Vib Digest 1999; 31(3): 187-194.

[28] Baharlou B, Leissa AW. Vibration and buckling of generally laminated composite plates with arbitrary edge conditions. Int J Mech Sci 1987; 29(8): 545-555.

[29] Aydogdu M, Timarci T. Vibration analysis of cross-ply laminated square plates with general boundary conditions. Compos Sci Technol 2003; 63(7): 1061-1070.

[30] Hu XX, Sakiyama T, Lim CW, Xiong Y, Matsuda H, Morita C. Vibration of angle-ply laminated plates with twist by Rayleigh-Ritz procedure. Comput Method Applied M 2004; 193(9-11): 805-823.

[31] Lee CR, Kam TY, Sun SJ. Free-vibration analysis and material constants identification of laminated composite sandwich plates. ASCE J Eng Mech 2007; 133(8): 874-886.

[32] Khorshidi K, Farhadi S. Free vibration analysis of a laminated composite rectangular plate in contact with a bounded fluid. Compos Struct 2013; 104: 176-186.

[33] Gradshteyn IS, Ryzhik IM. Table of integrals, series, and products $-7^{\text {th }}$ edition. Amsterdam: Elsevier, 2007.

[34] Washizu K. Variational methods in elasticity and plasticity. New York, NY: Pergamon Press, 1982.

[35] Ye T, Jin G, Ye X, Wang X. A series solution for the vibrations of composite laminated deep curved beams with general boundaries. Compos Struct 2015; 127: 450-465.

[36] Kant T, Swaminathan K. Analytical solutions for free vibration of laminated composite and sandwich plates based on a higher-order refined theory. Compos Struct 2001; 53(1): 73-85.

[37] Matsunaga H. Vibration and stability of cross-ply laminated composite plates according to a global higher-order plate theory. Compos Struct 2000; 48(4): 231-244.

[38] Reddy JN. A simple higher order theory for laminated composite plates. ASME J Appl Mech 1984; 51(4): 745752.

[39] Akavci SS. Buckling and free vibration analysis of symmetric and antisymmetric laminated composite plates on an elastic foundation. J Reinf Plast Compos 2007; 26(18): 1907-1919.

[40] Liew KM, Huang YQ, Reddy JN. Vibration analysis of symmetrically laminated plates based on FSDT using the moving least squares differential quadrature method. Comput Method Appl Mech Eng 2003; 192: $2203-2222$.

[41] Noor AK. Free vibration of multilayered composite plates. AIAA J 1973; 11(7): 1038-1039.

[42] Liew KM. Solving the vibration of thick symmetric laminates by Reissner/Mindlin plate theory and the p-Ritz method. J Sound Vib 1996; 198(3): 343-360.

[43] Jian WS, Akihiro N, Hiroshi K. Vibration analysis of fully clamped arbitrary laminated plate. Compos Struct 2004; 63(1): 115-122.

[44] Araújo dos Santos JV, Araújo AL, Mota Soares CM. Eigenfrequency analysis of completely free multilayered rectangular plates using a higher-order model and Ritz technique. Mech Compos Mater St 1998; 5(1): 55-79.

DOI: https://doi.org/10.1016/j.compstruc.2016.07.007 